\title{
A genome-wide transcriptome map of pistachio (Pistacia vera L.) provides novel insights into salinity-related genes and marker discovery
}

Maryam Moazzzam Jazi ${ }^{1}$, Seyed Mahdi Seyedi ${ }^{*}$, Esmaeil Ebrahimie ${ }^{2,3,4,5}$, Mansour Ebrahimi ${ }^{6}$, Gianluca De Moro ${ }^{7}$ and Christopher Botanga ${ }^{8}$

\begin{abstract}
Background: Pistachio (Pistacia vera L.) is one of the most important commercial nut crops worldwide. It is a salt-tolerant and long-lived tree, with the largest cultivation area in Iran. Climate change and subsequent increased soil salt content have adversely affected the pistachio yield in recent years. However, the lack of genomic/global transcriptomic sequences on P. vera impedes comprehensive researches at the molecular level. Hence, whole transcriptome sequencing is required to gain insight into functional genes and pathways in response to salt stress.

Results: RNA sequencing of a pooled sample representing 24 different tissues of two pistachio cultivars with contrasting salinity tolerance under control and salt treatment by Illumina Hiseq 2000 platform resulted in 368,953,262 clean 100 bp paired-ends reads $(90 \mathrm{~Gb})$. Following creating several assemblies and assessing their quality from multiple perspectives, we found that using the annotation-based metrics together with the length-based parameters allows an improved assessment of the transcriptome assembly quality, compared to the solely use of the length-based parameters. The generated assembly by Trinity was adopted for functional annotation and subsequent analyses. In total, 29,119 contigs annotated against all of five public databases, including NR, UniProt, TAIR10, KOG and InterProScan. Among 279 KEGG pathways supported by our assembly, we further examined the pathways involved in the plant hormone biosynthesis and signaling as well as those to be contributed to secondary metabolite biosynthesis due to their importance under salinity stress. In total, 11,337 SSRs were also identified, which the most abundant being dinucleotide repeats. Besides, 13,097 transcripts as candidate stress-responsive genes were identified. Expression of some of these genes experimentally validated through quantitative real-time PCR (qRT-PCR) that further confirmed the accuracy of the assembly. From this analysis, the contrasting expression pattern of NCED3 and SOS1 genes were observed between salt-sensitive and salt-tolerant cultivars.
\end{abstract}

Conclusion: This study, as the first report on the whole transcriptome survey of $P$. vera, provides important resources and paves the way for functional and comparative genomic studies on this major tree to discover the salinity tolerancerelated markers and stress response mechanisms for breeding of new pistachio cultivars with more salinity tolerance.

\footnotetext{
* Correspondence: Seyedi@nigeb.ac.ir

${ }^{1}$ Plant Biotechnology Department, National Institute of Genetic Engineering

and Biotechnology, Tehran, Iran

Full list of author information is available at the end of the article
} International License (http://creativecommons.org/licenses/by/4.0/), which permits unrestricted use, distribution, and reproduction in any medium, provided you give appropriate credit to the original author(s) and the source, provide a link to the Creative Commons license, and indicate if changes were made. The Creative Commons Public Domain Dedication waiver (http://creativecommons.org/publicdomain/zero/1.0/) applies to the data made available in this article, unless otherwise stated. 


\section{Background}

Pistachio belongs to the Anacardiaceae family and consists of at least 11 species, which $P$. vera is the only cultivated and economically important species [1]; the other species are mostly used as rootstocks for cultivation of $P$. vera. It has a long history of cultivation (3000-4000 years) in Iran [2]. Currently, Iran, United States, Turkey, and Syria are the major pistachio producers in the world; among them Iran is ranked first with an average 298, 838.67 tons of production from 1994 to 2014 [3]. Moreover, different parts of plant, including flower, leaf, seed and resins derived from stem, have pharmacological properties like antimicrobial, antioxidant and anti-inflammatory activities $[4,5]$.

In addition to its significant economic, nutritional, and medicinal values, $P$. vera is highly adaptable to abiotic stresses and considered as a tolerant species against drought and salt stresses, making it an ideal candidate for reforestation in arid and salinized zones [6, 7]. Although pistachio is categorized as a salt-tolerant glycophyte species, its yield is dramatically constrained under the high salinity conditions [8,9]. Due to global warming and drought in the last decade, forest area of wild pistachio in Iran has dramatically decreased, which has negative economic, environmental and social impacts. High temperature and low precipitation is responsible for the reduction of soil water storage, and increase in salt content, which is the most important limiting factor for the growth of rainfed trees. Therefore, there is an urgent need for the conservation and sustainable management of this major species to warrant prolonged growth and productivity periods. However, little attention has paid to this nut crop from the cellular molecular point of view that resulting in only 1329 expressed sequence tag (EST) sequences being in the National Center for Biotechnology Information (NCBI) (NCBI, search term "pistachio", as of December, 2016), and no reference genome. It has been very recently reported the genome survey of $P$. vera $\mathrm{cv}$. Siirt using Illumina sequencing to discover simple sequence repeats (SSR) within the assembled genome, which 59,280 SSR motifs were obtained and 206 SSRs applied to characterize $P$. vera cultivars and wild Pistacia genotypes. Based on this study, the pistachio genome is about $600 \mathrm{Mb}$ in size with a high heterozygosity rate that is probably owing to the dioecious mating system in this genus [10].

Most studies on $P$. vera have focused on limited SSR and random amplified polymorphic DNA (RAPD) markers development for germplasm characterization and sex determination [11-14], as well as the study of some physiological parameters in pistachio plants under drought and salt stresses [15-18]. However, there is no transcriptomic research and gene discovery at the large scale in this species, and in Anacardiaceae family only two RNA sequencing (RNA-seq) datasets are available from Mangifera indica [19] and P. chinensis [20]. In recent years, highthroughput RNA sequencing has emerged as an innovative and cost-effective tool for comprehensive transcriptome profiling in model and non-model plants [21]. While transcriptome analysis would be carried out using mappingbased approaches for model plants, deep sequencing followed by creating a high quality de novo assembled transcriptome is of first priority for downstream analysis in non-model plants $[22,23]$. Most de novo transcriptome assemblers attempt to recognize the contiguous sequences by generating a de-Bruijn graph, which the k-mer length is one of the key factors that affect the assembly output. The optimal k-mer length for a given assembly relies on sequencing depth and genome/transcriptome complexity [21]. However, obtaining the high quality transcriptome assembly requires the assembly evaluation from multiple perspectives [24].

The objective of this study is to (1) create a robust pooled transcriptome assembly from leaf, stem, and root of $P$. vera under control and salinity conditions to provide a reference sequence, (2) compare the three well-known transcriptome assemblers, including CLC Genomics Workbench, Trinity and SOAPdenovo-Trans with single and multiple k-mer length using several length- and annotation-based metrics, (3) functionally annotate the reconstructed transcripts, (4) examine the biological pathways and genes related to salt stress, (5) develop the SSR markers for future marker-based studies, and (6) analyze selected salt-stress responsive genes using qRT-PCR.

The present study, as the first report on the transcriptome sequencing of $P$. vera, will not only provide plenty of informative data, but also paves the way for functional and comparative genomic studies on this species, as well as, other related species. Owing to similar signal transduction pathways and plant response mechanisms among salinity, cold, and drought stresses, the resulting genomewide transcriptome map of pistachio under salt stress is a valuable platform for upcoming RNA-seq analysis of gene expression of this major species under these abiotic stresses. Finally, since root, stem and leaves of $P$. vera were sampled and sequenced at the high sequencing depth, the obtained annotated assembly will definitely facilitates RNA-seq analysis of any above tissues in future studies.

\section{Methods}

\section{Plant materials and salinity treatment}

In order to select the salt-sensitive and salt-tolerant pistachio cultivars, the surface-sterilized seeds of five cultivars, including $P$. vera L. cv. Sarakhs, Badami-zarand, Ghazvini, Akbari and Kaleghuchi as the main indigenous cultivars of Iran were obtained from Iranian Pistachio Research Institute, and grown in boxes containing Hoagland's nutrient solution $(\mathrm{pH}=5.8)$ with 16 -h light $/ 8$-h 
dark photoperiods for 6 weeks. The 6-week-old plants were exposed to salt stress by adding $250 \mathrm{mM} \mathrm{NaCl}$ to the hydroponic culture medium. In order to avoid osmotic shock, salt was added incrementally up to the final salt concentration. The nutrient solution was renewed every 3 days. The experiment was performed with 30 plants per cultivar with three biological replicates. For cultivar selection, plant survival rate was measured after 8 days of exposure to salinity. Based on the results, Sarakhs and Ghazvini cultivars were determined as salt-sensitive and salt-tolerant cultivars with $24.4 \%$ and $65 \%$ of survival percentage after 8 days of salt stress, respectively (Additional file 1). For more assessment, sodium and potassium content as the main elements of salinity stress and Malondialdehyde (MDA), as the cell membrane lipid peroxidation index, were measured in roots of all cultivars on the 8th day after salt treatment. Based on statistical analysis, there were significant differences ( $p$-value $<0.05)$ among cultivars, specifically between Sarakhs and Ghazvini as we expected. The lowest level of sodium and the highest level of potassium were accumulated in the Ghazvini roots while the opposite pattern was observed for roots of Sarakhs. Similarly, in contrast to Sarakhs, the MDA content was the lowest in the Ghazvini roots (Additional file 1).

For transcriptome sequencing, the surface-sterilized seeds of selected rootstocks, P. vera L. cv. Sarakhs and Ghazvini as the most salt-sensitive and salt-tolerant cultivars, respectively, were grown and subjected to salt stress as above mentioned. We used 7 plants for each salt-sensitive and salt-tolerant cultivars with three biological replicates (in total, 21 plants for each cultivar) at each time point, including $0,6,24$, and $48 \mathrm{~h}$ post salt treatment. The leaves, stems, and roots of 21 plants were separately harvested at each time point and immediately frozen in liquid nitrogen. For RNA extraction, we pooled 21 samples of each tissue at each time point together as one sample. Total RNA was finally isolated from 24 samples, containing two cultivars, three types of tissue, and four time points.

\section{RNA extraction, library preparation and RNA sequencing}

The total RNA was separately isolated from $200 \mathrm{mg}$ of harvested tissues (in total, 24 samples) using a modified CTAB (cetyltrimethyl ammonium bromide) method [25]. The purity and quantity of each RNA sample were determined by NanoDrop $2000{ }^{ }{ }^{\mathrm{m}}$ micro-volume spectrophotometer (Thermo Scientific, Waltham, MA, USA), and gel electrophoresis. The final quality assessment was performed using Agilent Bio Analyzer 2100 prior to deep sequencing. Only RNA samples with RIN (RNA integrity number) of more than 8 were used for further processing. Extracted RNA samples were treated with RNase-free DNase I to remove the probable DNA contamination.
Pooled RNA sample was made for comprehensive transcriptome sequencing through mixing an equal amount of extracted RNA $(1 \mu \mathrm{g})$ from each sample. Library preparation from the pooled RNA sample representing 24 tissues of two pistachio cultivars under normal and stress conditions was performed as outlined in Illumina's TruSeq Stranded mRNA Sample Prep Kit (Illumina Inc., U.S.A). The cDNA library was sequenced on the one lane of Illumina Hiseq 2000 platform as $2 \times 100$ run according to the manufacturer's instructions. The raw sequencing reads produced in this study have been deposited at NCBI in the Short Read Archive (SRA) database under the accession number SRX1880621.

\section{Read pre-processing}

Following the assessment of raw reads quality using FastQC tool (http://www.bioinformatics.babraham.ac.uk/ projects/fastqc/), the reads were pre-processed to eliminate low quality bases and probable contaminants. We used CLC genomics workbench software (v7.5, CLCBio, Qiagen) for removing low quality bases (Phred score cutoff $=20$ ) and adapter sequences. The probable ribosomal-derived reads were also filtered from the sequencing data using SortMeRNA tool prior to the assembly step [26].

\section{De novo transcriptome assembly}

De novo transcriptome assembly was conducted using two strategies through three well-known assemblers, Trinity (v2014-07-17) [27], SOAPdenovo-Trans (v1.03) [28], and CLC genomics workbench (v7.5) (http://www.clcbio.com), which have been developed for the assembly of short reads by de Bruijn graph algorithm [29]. In the first strategy, clean reads were assembled to the contigs with k-mer of 25 using Trinity, SOAPdenovo-Trans and CLC programs with default settings except for Trinity, in which the strand-specific as well as in silico read normalization options were implemented. In the second strategy, different assemblies were made with the k-mer lengths of $25,29,33,37,41,45,49,51,55,59$, and 63 using SOAPdenovo-Trans and CLC tools; no-scaffold option was set during the assembly to avoid the strings of N's connecting separate contigs. Contigs shorter than $300 \mathrm{bp}$ were discarded from all the de Bruijn graph-based assemblies. All 9 individual k-mer assemblies resulted from SOAPdenovo-Trans and CLC tools were separately combined into a single merged assembly, which was further processed by CD-HIT-EST tool (v4.6.1-2012-08-2, 30] at 99\% identity level (cd-hit-est. -c $0.99-n 8-r$ ) in order to reduce redundancy and remove the identical fragments. The processed assembly was exposed to CAP3 program [30] with default settings to perform a meta-assembly and generate longer and more complete consensus sequences. The outputs of CAP3, contigs and singletons, were pooled 
together to form a final assembly. An overview of our workflow is illustrated in Fig. 1.

\section{Transcriptome assembly quality evaluation}

The output of each assembly was interrogated using several quality metrics including, length- and annotationbased parameters. Contig length information such as N50 length, total contig number, average, and maximum contig length were assessed for each assembly using CLC software and ordinary Perl scripts. Raw reads were also aligned back to the each assembly by CLC program with the setting of mismatch/insertion/deletion costs at $2 / 3 / 3$ and length fraction/similarity to $0.75 / 0.90$ and the percentage of mapped back reads to transcripts were assessed. The completeness of each assembly was estimated using the Core Eukaryotic Genes Mapping Approach (CEGMA) tool [31]. Moreover, all protein sequences of Citrus sinensis, as the closely related species to $P$. vera, were downloaded from Phytozome (v10.3) and utilized for homology search. Each assembly was compared with C. sinensis proteome using BLASTX with the e-value cutoff of 1 e- 5 retaining only top match for each query sequence. Likewise, assembled transcripts were reverse annotated through the citrus dataset by TBLASTN with the same e-value cutoff using NCBI-BLAST software $(\mathrm{v} 2.2 .30+)$ [24]. Then, the number of unique proteins

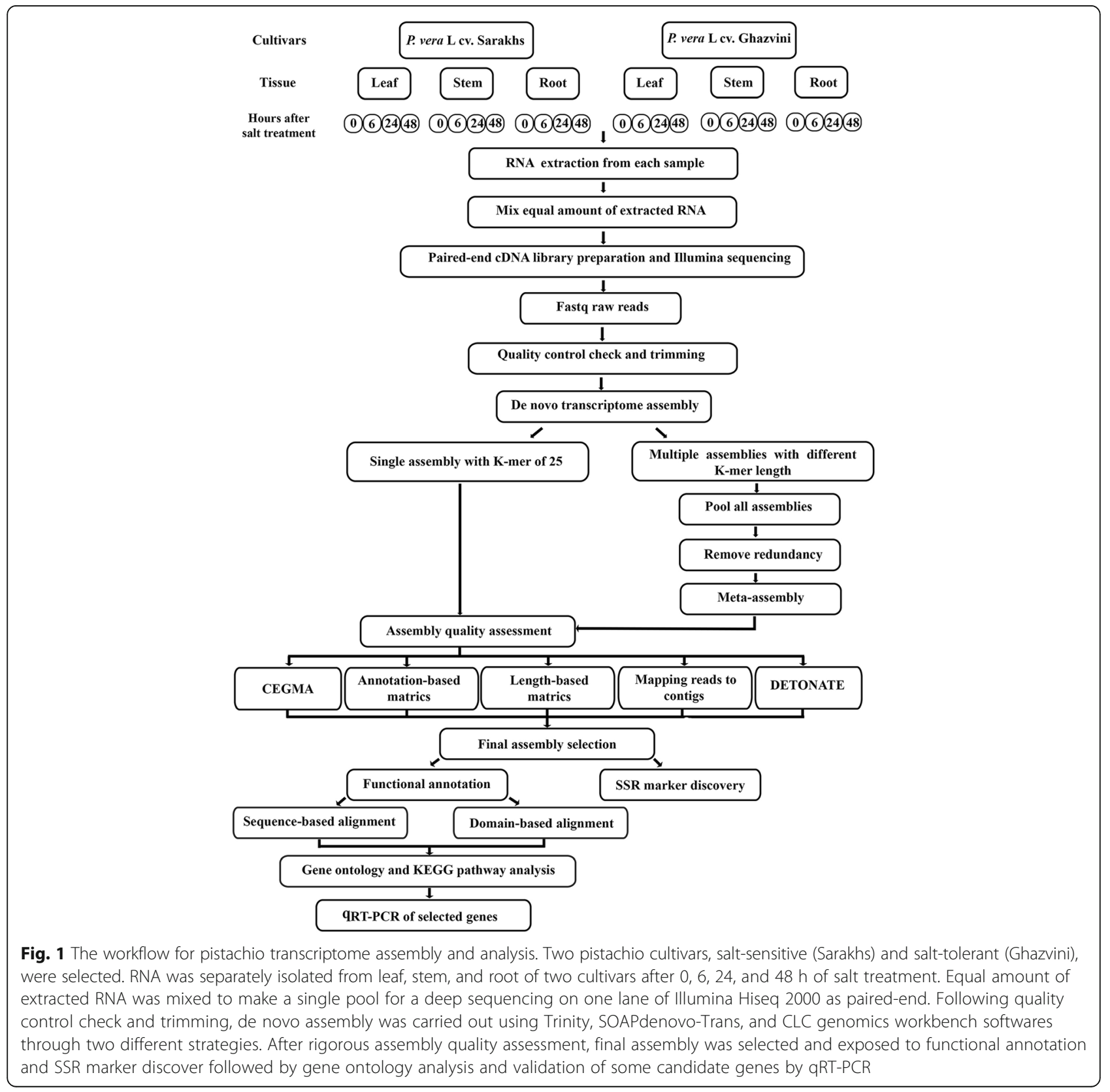


found in the BLASTX, the number of reciprocal best hits $(\mathrm{RBH})$ and the ortholog hit ratio (OHR) were computed for each assembly as described by O'Neill and Emrich [24]. Further, the RSEM-EVAL tool from DETONATE (DE novo TranscriptOme rNaseq Assembly with or without the Truth Evaluation) package was employed for different assemblies assessment [32] (Fig. 1).

\section{Functional annotation of the assembled pistachio transcripts} Prior to annotation, TransDecoder program (v2.1.0) (https://transdecoder.github.io) was used in order to predict the protein-coding open reading frames (ORFs). To recognize homologous proteins, all deduced protein sequences resulted from Trinity assembly were aligned against the NR (non-redundant), UniProt (Swiss-Prot and TrEMBL), TAIR10 (the Arabidopsis information resource), and KOG (eukaryotic orthologous groups) protein databases using locally installed NCBI-BLAST software (v2.2.30+) with the e-value threshold of 1e-5. To annotate transcripts with GO terms, the best blast hit from the NR database was imported to Blast2GO software (v 3.2). Likewise, the predicted protein sequences were searched against Pfam, ProDom, ProSiteProfiles by InterProScan software (v5.14-53.0) [33] for retrieving conserved domains/motifs and corresponding GO terms. Additionally, KEGG (kyoto encyclopedia of genes and genomes) pathway analysis was conducted using the Automatic Annotation Server (KAAS; http://www.genome.jp/ $\mathrm{kegg} / \mathrm{kaas} /$ ) with single-directional best hit (SBH) method for KEGG orthology (KO) assignments and pathway mapping.

\section{Candidate stress-responsive genes identification}

To identify the potential stress-responsive genes in the $P$. vera transcriptome, all final assembled transcripts were searched against the genes involved in various abiotic stresses (salt, drought, and cold) [34, 35] using BLASTX with the e-value cutoff of 1e-5.

\section{Quantitative real-time PCR}

The selected transcripts related to salinity-responsive genes were chosen for validation by quantitative real-time PCR (qRT-PCR). All required primers were designed using Oligo 7 software (Additional file 2); the elongation factor 1 alpha $(E F 1 \alpha)$ was applied as a reference gene [9]. qRT-PCR was carried out using SYBR Green real-time PCR master mix (Ampliqon, Denmark). The reaction mixture contained $1.0 \mu \mathrm{L}$ of diluted cDNA sample, $0.5 \mu \mathrm{L}$ of each of the forward and reverse primers $(10 \mu \mathrm{M})$ and $10 \mu \mathrm{L}$ real-time master mix with a final volume of $20 \mu \mathrm{L}$. The cycling conditions were as follows, initial activation of DNA polymerase at $95^{\circ} \mathrm{C}$ for $10 \mathrm{~min}$, followed by 40 cycles $94{ }^{\circ} \mathrm{C}$ for $30 \mathrm{~s}, 63{ }^{\circ} \mathrm{C}$ for $30 \mathrm{~s}$ and $72{ }^{\circ} \mathrm{C}$ for $20 \mathrm{~s}$. Each sample was examined in three biological replications. A melting-curve analysis was $65{ }^{\circ} \mathrm{C}$ to $95{ }^{\circ} \mathrm{C}$, with fluorescence measured every $0.5{ }^{\circ} \mathrm{C}$ at the end of each reaction to further confirm the specificity of the primer pairs. The relative gene expression was computed by Pfaffl formula [36] embedded in REST 2009 software.

\section{SSR marker discovery}

The SSR Locator V.1 software was used for SSR identification, primer design and virtual PCR [37]. All type of SSRs, from dinucleotide to hexanucleotide repeats were searched on the transcriptome assembly generated by Trinity. The minimum repeat number of six for dinucleotide and five for all other repeats were specified for this analysis.

\section{Results and discussion}

\section{Pistachio transcriptome sequencing}

High-throughput sequencing technology has provided an excellent opportunity for transcriptome survey in non-model plant species, like $P$. vera. Here, a total of $394,579,946$ million raw reads (90 $\mathrm{Gb}$ of data) as pairedend $(\mathrm{PE})$ generated from a pooled RNA sample. For making a robust reference transcriptome that cover a wide range of transcripts, equal amount of extracted RNA from leaf, stem, and root of salt-tolerant and -sensitive cultivars at $0,6,24$, and $48 \mathrm{~h}$ post salt treatment was pooled and sequenced on one lane of Illumina Hiseq 2000 platform. After trimming of the low quality bases and adapters as well as the removal of rRNA, a total of $368,953,262$ million clean PE reads with an average quality score of 38 were reminded for transcriptome assembly (Table 1). rRNA is the most abundant transcript within total RNA, causing a major challenge during transcriptome assembly by decreasing the sequencing depth for informative mRNAs [38]. While we used poly $\mathrm{A}^{+}$selection method for mRNA enrichment, 3.7\% of reads were still aligned to ribosomal RNA that properly removed before doing de novo assembly. The resulting data and subsequent analyses constitute an unprecedented look into the $P$. vera transcriptome at a single nucleotide resolution.

Table 1 Basic statistics of pistachio sequencing reads obtained from Illumina HiSeq-2000

\begin{tabular}{ll}
\hline Parameter & Value \\
\hline Number of raw reads & $394,579,946$ \\
Reads average length before trimming (bp) & 100 \\
Reads average length after trimming (bp) & 98 \\
Number of ribosomal RNA reads & $14,333,680$ \\
Number of reads after trimming & $368,953,262$ \\
Average quality score & 38 \\
GC content (\%) & 44 \\
\hline
\end{tabular}


Generation of a de novo-assembled, genome-wide transcriptome map of $P$. vera and assembly statistics In order to construct a high quality assembly, several transcriptome assemblies were created using three stateof-the-art de Bruijn graph-based assemblers, including CLC Genomics Workbench, SOAPdenovo-Trans, and Trinity, which accept multiple k-mer and single k-mer value, respectively. As Trinity efficiently produced a transcriptome assembly with its default k-mer of 25, we selected this k-mer length across three assemblers to compare them at the single k-mer size (the first strategy). While the contig number, length distribution, and length-weighted medians (N50 and N90) were comparable between assemblies produced by SOAPdenovoTrans and CLC softwares, the highest number of contigs $(144,103)$, of which $59,727(41.4 \%)$ were at least $1 \mathrm{~kb}$ in length, were generated by Trinity (Table 2). With the constant k-mer of 25, a considerable difference was found in average contig length and N50 value among assemblies. Again, the highest average of transcript length (1139 bp) and N50 size (1679 bp) were obtained from Trinity assembly (Table 2). Due to the non-uniform nature of transcriptome assembly, low and high abundant transcripts might theoretically represent at smaller and larger k-mer size, respectively [39]. Therefore, in the second strategy, we used the k-mer size of 25-63 with the step size of 4 for making multiple k-mer assemblies through SOAPdenovo-Trans and CLC. All generated contigs by two assemblers with various $\mathrm{k}$-mer lengths were independently merged and fed to cd-hit-est. program for removing redundant contigs. This tool is a popular clustering program based on the greedy incremental clustering method, which groups nucleotide sequences of a given dataset into clusters that meet a userdefined similarity threshold and the longest sequences of each cluster are selected as representatives [40]. This step significantly reduced the contig total number from 602,146 to 165,895 for SOAPdenovo-Trans assembly and 584,686 to 169,120 for CLC one. The non-redundant contigs were then subjected to CAP3 software to compute the overlaps and produce a consensus assembly. CAP3 is an efficient sequence assembler that connects highly similar overlapping sequences and thus further reduces transcript redundancy [30]. The resulting contigs and singletons obtained from CAP3 were collapsed together for generating a final merged assembly. As shown in Table 2, the contig

Table 2 Transcriptome assembly quality evaluation metrics

\begin{tabular}{|c|c|c|c|c|c|}
\hline Parameters & CLC & SOAPdenovo-Trans & Trinity & $\begin{array}{l}\text { Merged assembly } \\
\text { (SOAPdenovo-Trans) }\end{array}$ & Merged assembly (CLC) \\
\hline Number of contigs & 83,390 & 85,091 & 144,103 & 93,865 & 90,632 \\
\hline Average transcript length (bp) & 787 & 695 & 1139 & 885 & 945 \\
\hline Minimum transcript length (bp) & 300 & 300 & 300 & 300 & 300 \\
\hline Maximum transcript length (bp) & 12,370 & 12,097 & 13,939 & 13,329 & 12,167 \\
\hline N50 (bp) & 909 & 788 & 1679 & 1300 & 1450 \\
\hline N90 (bp) & 392 & 361 & 489 & 397 & 425 \\
\hline Percentage of contig $\geq 1 \mathrm{~kb}$ & 20.92 & 16.47 & 41.44 & $30 \%$ & $33.83 \%$ \\
\hline $\begin{array}{l}\text { Percentage of mapped back reads to } \\
\text { assembly }\end{array}$ & 88.51 & 83.81 & 97.49 & 93.54 & 96.98 \\
\hline Percentage of mapped reads in pairs & 78.23 & 70.71 & 89.93 & 83.67 & 87.76 \\
\hline $\begin{array}{l}\text { Percentage of mapped broken paired } \\
\text { reads }\end{array}$ & 10.28 & 13.1 & 7.86 & 9.54 & 9.22 \\
\hline $\begin{array}{l}\text { Percentage of complete cores proteins by } \\
\text { CEGMA analysis }\end{array}$ & 79.03 & 69.35 & 97.98 & 95.16 & 94.35 \\
\hline $\begin{array}{l}\text { Percentage of partial cores proteins by } \\
\text { CEGMA analysis }\end{array}$ & 93.15 & 93.15 & 99.6 & 97.74 & 98.79 \\
\hline $\begin{array}{l}\text { The number of unique proteins found in } \\
\text { blastx }\end{array}$ & 20,782 & 20,872 & 25,065 & 20,789 & 20,911 \\
\hline $\begin{array}{l}\text { The number of unique contigs hit by } \\
\text { proteins in the tblastn }\end{array}$ & 44,479 & 44,503 & 44,807 & 44,302 & 44,803 \\
\hline $\begin{array}{l}\text { The number of unique contigs with } \\
\text { reciprocal best hits }\end{array}$ & 13,730 & 13,724 & 15,690 & 14,003 & 14,101 \\
\hline $\begin{array}{l}\text { The number of unique contigs with } \\
\text { orthologue hit ratio of } 0.8-1\end{array}$ & 5963 & 7249 & 12,903 & 7750 & 8423 \\
\hline RSEM-EVAL score & $-19,590,755,435$ & $-22,457,309,826$ & $-11,702,493,371$ & $-14,998,853,445$ & $-13,665,328,361$ \\
\hline
\end{tabular}

Transcriptome assembly evaluation metrics for single $25 \mathrm{k}$-mer assemblies generated by CLC genomics workbench, SOAPdenovo-Trans and Trinity as well as the merged assembly with k-mer length ranging from 25 to 63 with the step size of 4 
average length and N50 value were notably increased in the merged assemblies compared to the corresponding single assemblies (Table 2). These results are consistent with previous reports of the higher performance of merged assemblies in Crocus sativus [41].

\section{Assessing transcriptome assembly quality}

Although parameters like, N50 and average contig length are commonly used statistics for genome assembly evaluation, they may have little informative value for transcriptome assembly evaluation [42]. In fact, N50 measures the continuity of contigs but not their accuracy. Similarly, the larger N50 length or smaller contig number do not necessarily imply a better de novo transcriptome assembly $[24,43]$. Hence, in addition to assessing the basic lengthbased parameters, the assembly quality was evaluated using a number of more stringent criteria as explained below (Fig. 1).

\section{Mapping reads to the assembled transcripts}

The percentage of reads mapped back to assembled transcripts (RMBT) is considered to examine the assembly completeness, implicating the read inclusion amount to construct the assembly [44]. Ideally, the high quality assembly should have the high RMBT percentage. The high RMBT in our study was obtained from the Trinity and merged assemblies, followed by the single k-mer assembly created by CLC and the assembly made by SOAPdenovo-Trans that had the lowest mapped reads (Table 2). Our results indicate that Trinity at the single k-mer length utilizes the majority of sequencing reads to construct the assembly, in contrast to other programs and strategies. Additionally, as compared with other assemblies, Trinity assembly had the highest percentage of mapped reads in pairs and the lowest percentage of broken paired reads, the paired reads that each mate of a read mapped on two different assembled transcripts (Table 2), which may implicate the lower fragmented transcripts produced by Trinity.

\section{Identification of widely conserved eukaryotic genes}

CEGMA program conducts a similarity search of assembly against the subset of KOG database consisting 248 highly conserved proteins from a wide range of eukaryotes [31]. These proteins mostly belong to housekeeping genes group and were expected to be expressed in many tissues [45]. We incorporated it in our pipeline for evaluating the assembly completeness, the number of proteins represented in each assembly is shown in Table 2. CEGMA can also discriminate between full-length (complete) and partial predicted core proteins. While CEGMA analysis recognized 243 out of 248 core proteins (97.98\%) as complete (70\% alignment length of assembled transcript with core protein) within the Trinity assembly, the 79.03 and $69.35 \%$ of full-length core proteins were detected for the single k-mer assembly created by CLC and SOAPdenovo-Trans softwares, respectively (Table 2). Comparing to these single $\mathrm{k}$-mer assemblies, the merged assemblies covered the more number of core proteins, referring to the higher transcript number came from this approach. The percentage of partial core proteins was higher than complete ones in all cases (Table 2), offering some genes were split on the several contigs.

\section{Comparisons with orthologs}

Moreover, we used several annotation-based metrics for evaluating the transcriptome assembly quality as described by O'Neil and Emrich [24]. For this assessment, BLASTX and TBLASTN algorithms were applied for comparison of the assembled transcriptome with Citrus sinensis proteome, as the closest reference species. According to a phylogenetic study of mango ( $M$. indica) chloroplast DNA, C. sinensis was closely related to $M$. indica [46], which is belongs to the Anacardiaceae family. As presented in Table 2, the number of unique $C$. sinensis proteins found in the BLASTX search was comparable for all assemblies, except for the Trinity assembly with the highest percentage of citrus proteins (Table 2). Overall, about $45-54 \%$ of C. sinensis proteins were covered by each assembly, probably due to species divergence and the lack of complete gene expression in our experimental conditions. To identify the reciprocal best hit $(\mathrm{RBH})$ and actual ortholog between $P$. vera and $C$. sinensis, reverse annotation was performed using TBLASTN. The number of RBH was computed based on the BLASTX and TBLASTN outputs for each assembly. As a result, the highest number of RBH $(15,690)$ was produced by Trinity assembly followed by merged and single assemblies (Table 2). It is worth noting that the RBH level depends on the evolutionary divergence between the species of interest and related reference [47]. Considering the fact that the high quality transcriptome assembly would reconstruct more full-length transcripts, the OHR was also estimated for the various assemblies to determine the contig content assembled into an ortholog sequence $[48,49]$. Since assessing transcript integrity relies on the contig and its best hit are ortholog, only contigs with $\mathrm{RBH}$ were used for $\mathrm{OHR}$ calculation. Therefore this metric is conservative as parts of orthologs may be represented by contigs that are not the reciprocal best hit [50]. Our findings showed that the highest percentage of full length transcripts was constructed by Trinity, so that the ortholog hit ratio for 12,904 contigs $(82.24 \%$ ) with $\mathrm{RBH}$ was $0.8-1$, referring to near full length or full length transcript (Table 2). This parameter was computed by comparing the length of assembled contigs relative to the length of known ortholog sequences, hence the OHR of 1 considered as a full length transcript [24]. As the OHR 
distribution plot illustrates (Fig. 2), Trinity assembly followed by the merged assemblies produced significantly more full-length transcripts than the single assemblies generated by SOAPdenovo-Trans and CLC tools. However, gaining partial transcripts with OHR of less than 0.8 represented by all assemblies was unavoidable, implying that the full-length transcript construction is a challenge during de novo transcriptome assembly, particularly for non-model organisms. Although, the length-based parameters were significantly improved in the merged assembly as compared with the single assembly, the number of unique proteins found in BLASTX and the number of unique contigs with reciprocal best hits were slightly better in these assemblies. Hence, using the annotation-based metrics together with the length-based parameters allows an improved assessment of the transcriptome assembly quality, compared to the solely use of the length-based parameters.

\section{Assembly evaluation using DETONATE}

RSEM-EVAL is a reference-free approach bundled with DETONATE package that was recently developed for transcriptome assembly evaluation [32]. In this study, RSEM-EVAL score was calculated for each transcriptome assembly as well. This score is based on a probabilistic model that accepts the clean reads and assembly as input [51]. Assemblies with the higher score are considered to be better. As presented in Table 2, the RSEM-EVAL score of merged assemblies generated by CLC and SOAPdenovo-Trans softwares were slightly different, but higher than the corresponding single assemblies. Among all assemblies, Trinity assembly with the highest score was the best one (Table 2), revealing that this program performed more accurately on individual contigs level than CLC and SOAPdenovo-trans. Overall, according to all above mentioned evaluation criteria, we adopted Trinity output for further analyses.
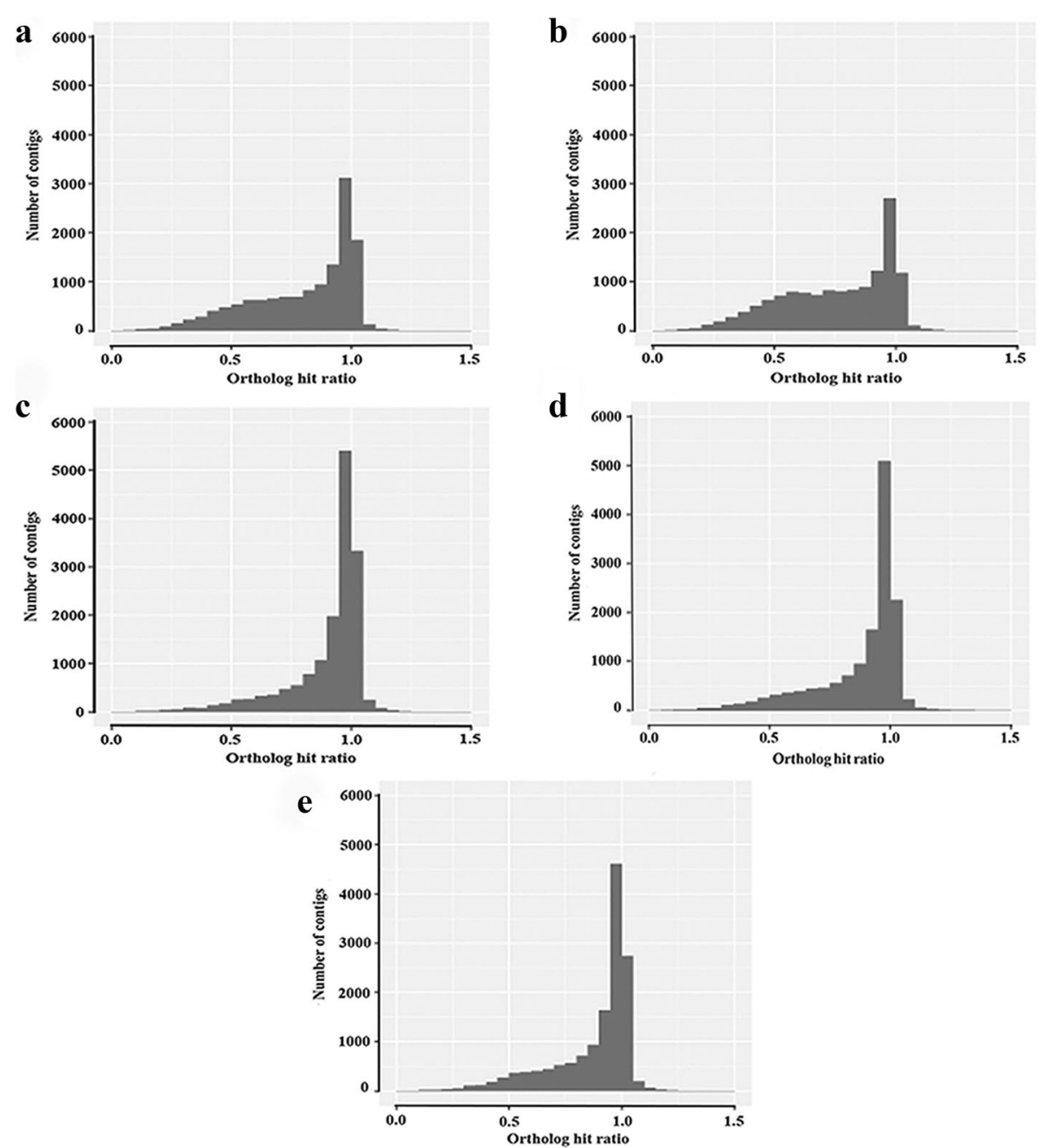

Fig. 2 Ortholog hit ratio of assembled transcripts for various assemblies. a Single assembly, CLC genomics workbench. b Single assembly, SOAPdenovo-Trans. c Single assembly, Trinity. d Merged assembly, CLC genomics workbench. e Merged assembly, SOAPdenovo-trans 


\section{Functional annotation of $P$. vera transcriptome}

The integration of transcriptome assembly with functional annotation is the main aspect of mining transcriptomic data in a non-model organism. Here, to predict the function of assembled pistachio transcripts, similarity search was performed at two levels, sequence-based and domain-based alignments. To facilitate the procedure, transcripts with open reading frames (ORF) were extracted from the Trinity assembly using TransDecoder program. Out of 144,103 transcripts, 94,826 (65.8\%) potential protein sequences were obtained, of which 52,971 (55.86\%) sequence were reported as full length (Table 3 ). For sequence-based annotation, all deduced protein sequences were aligned against the NR database using BLASTP algorithm and the best blast hit imported to Blast2GO software for downstream analysis. Out of 94,826 sequences, 84,117 (88.7\%) had a significant blast hit with the e-value threshold of 1e-5 (Table 3 and Additional file 3). Although we applied an e-value cutoff of 1e-5 for homology search analysis, the e-value for the major fraction of matched sequences ranged between 1e-10 and 1 e-180 as illustrated in Fig. 3a. Similarity distribution demonstrated that the maximum number of matched sequences have $88 \%$ positive alignment length (Fig. 3b), the lack of best blast hits with less than 35\% sequence similarity with respect to query implied the appropriate homology with the query. In terms of species distribution, the majority of annotated sequences showed the highest homology to sequences from Citrus sinensis (39.7\%), followed by Citrus clementina (18\%), Theobroma cacao (7\%) and Vitis vinifera (4\%) (Fig. 3c). It has been reported that $M$. indica and $C$. sinensis are phylogenetically related species [46]. P. vera and $M$. indica, belong to the Anacardiaceae family, suggesting that the pistachio transcriptome achieved in the present study was properly annotated. Meanwhile, only 161 sequences in our assembly showed best matches with $M$. indica, which could be due to little publicly available

Table 3 Functional annotation summary of $P$. vera transcriptome

\begin{tabular}{ll}
\hline Parameter & Number of sequence \\
\hline Potential protein sequences & 94,826 \\
Annotated sequence against NR & 84,117 \\
Annotated sequence against UniProt & 82,180 \\
Annotated sequence against TAIR10 & 73,276 \\
Annotated sequence against InterProScan & 66,029 \\
Annotated sequence against KOG & 33,288 \\
Sequences matching all five databases & 29,119 \\
Sequence annotated with GO terms & 68,539 \\
Sequences assigned with EC numbers & 21,598 \\
Annotated sequence against KEGG & 34,924 \\
\hline
\end{tabular}

sequence information for this species. In order to obtain further descriptions and improve the pistachio annotation, the current assembly was blasted against two other protein databases, including UniProt (Swiss-Prot and TrEMBL) and TAIR10. Compared to UniProt, a total of $82,180(86.6 \%)$ significant best blast hits were returned with the e-value threshold of 1e-5 (Table 3). In the case of TAIR10, comprising a complete reference genome and well-annotated sequences for Arabidopsis thaliana, 73,276 (77.27\%) sequences displayed significant homology with this database (Table 3).

Considering that the proteins with little similarity at the sequence level can share conserved domains, we carried out a domain-based annotation by searching the candidate protein sequences against Pfam, ProDom, and ProSiteProfiles databases using InterProScan program. Results revealed that a total of 66,029 sequences $(69.77 \%)$ were categorized into 4235 domains/families. Based on the number of $P$. vera transcripts contained in each InterProScan domains/families, the InterPro domains/families ranked and listed the top 30 abundant domains/families in Table 4. The most represented protein region was a Pentatricopeptide repeat (PPR) (IPR002885) with 14,926 assigned sequences. Consistent with our findings, it has been reported that proteins with PPR site constitute one of the largest protein families in plants with 450 members in Arabidopsis thaliana and more than 600 members in Oryza sativa $[52,53]$. PPR proteins are mostly targeted to mitochondria or chloroplasts, where they are involved in organelle gene expression via RNA processing, splicing, stability, editing, translation, and plant response to abiotic stresses [52, 54]. Other commonly occurring domains/ sites in the $P$. vera transcriptome were protein kinase domains (IPR000719), leucine-rich repeats (IPR001611), and WD40 repeats (IPR001680). Protein kinase domain and its subclass, serine-threonine/tyrosine-protein kinase were recognized to regulate the majority of cellular processes, including signal transduction, differentiation, as well as, cell growth and development. Leucine-rich repeat regions are known to have a repeating stretch of 20-29 amino acids that form an $\alpha / \beta$ horseshoe fold involved in the formation of protein-protein interactions [55]. Similarly, WD40 repeat act as a site for protein-protein interaction and WD40-containing proteins serve as platforms for the assembly of protein complexes or mediators of transient interplay among other proteins [56].

The KOG database built on phylogenetic classification of proteins encoded in 66 complete genomes, including bacteria, plants, and animals. Each KOG cluster consists of a protein or a group of proteins from at least 3 different eukaryotic lineages further clustered based on their function [57]. We aligned the translated assembly to the KOG database in order to predict and classify their possible functions. KOG classification of our assembled 

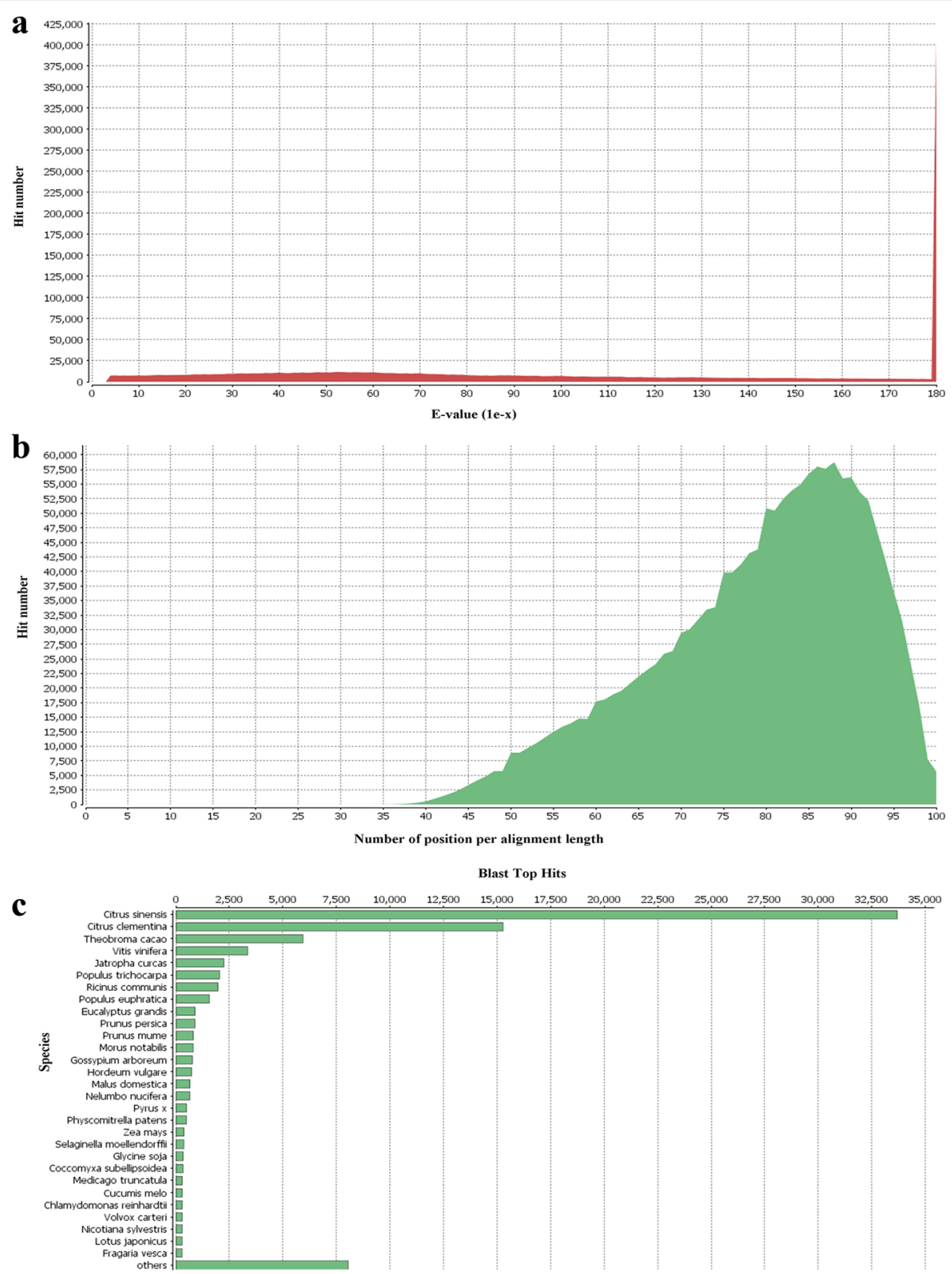

Fig. 3 Graphical representations of functional annotations in P. vera transcriptome. a E-value distribution graph. $\mathbf{b}$ Similarity distribution graph. c Top-hit species distribution graph

transcripts revealed that 33,288 protein sequences were clustered into 25 functional categories under four larger groups (metabolism, cellular processes and signaling, information storage and processing, and poorly characterized) (Additional file 4). Among the different KOG categories, the highest number of sequences were assigned to the "signal transduction mechanisms" category (4902, 14.7\%), followed by "general function prediction only" (4108, $12.34 \%$ ) and "posttranslational modification, protein turnover, chaperones" (3832, 11.5\%). In the metabolism group, "Carbohydrate transport and metabolism" (5.9\%), "amino acid transport and metabolism" (5.4\%), and "Intracellular trafficking, secretion, and vesicular transport" (5.3\%) were also highly represented. However, 1748 (5.3\%) of total protein sequences annotated with KOG database were classified as "function unknown", suggesting that $P$. vera is almost a phylogenetically distant species compared to those present in the KOG database. Taken together, 29,119 sequences exhibited the similarity to proteins in all of the five public databases, including NR, UniProt, TAIR10, KOG and InterProScan (Fig. 4). 
Table 4 List of the top-hit 30 InterPro domains in P.vera transcriptome

\begin{tabular}{|c|c|c|}
\hline InterPro domain & Description & $\begin{array}{l}\text { Number of } \\
\text { sequence }\end{array}$ \\
\hline IPR002885 & Pentatricopeptide repeat & 14,926 \\
\hline IPR000719 & Protein kinase domain & 50,94 \\
\hline IPR001611 & Leucine-rich repeat & 3983 \\
\hline IPR001680 & WD40 repeat & 2556 \\
\hline IPR000504 & RNA recognition motif domain & 1828 \\
\hline IPR019734 & Tetratricopeptide repeat & 1235 \\
\hline IPR002048 & EF-hand domain & 1185 \\
\hline IPR001245 & $\begin{array}{l}\text { Serine-threonine/tyrosine-protein } \\
\text { kinase catalytic domain }\end{array}$ & 1176 \\
\hline IPR001841 & Zinc finger, RING-type & 1155 \\
\hline IPR020683 & Ankyrin repeat-containing domain & 1055 \\
\hline IPR017986 & WD40-repeat-containing domain & 9,30 \\
\hline IPR002110 & Ankyrin repeat & 883 \\
\hline IPR018108 & Mitochondrial substrate/solute carrier & 839 \\
\hline IPR001650 & Helicase, C-terminal & 808 \\
\hline IPR003439 & $\mathrm{ABC}$ transporter-like & 719 \\
\hline IPR001128 & Cytochrome P450 & 692 \\
\hline IPR002182 & NB-ARC & 632 \\
\hline IPR001810 & F-box domain & 562 \\
\hline IPR013026 & $\begin{array}{l}\text { Tetratricopeptide repeat-containing } \\
\text { domain }\end{array}$ & 538 \\
\hline IPR000571 & Zinc finger, CCCH-type & 517 \\
\hline IPR001932 & PPM-type phosphatase domain & 508 \\
\hline IPR017930 & Myb domain & 493 \\
\hline IPR011598 & $\begin{array}{l}\text { Myc-type, basic helix-loop-helix } \\
\text { (bHLH) domain }\end{array}$ & 489 \\
\hline IPR000048 & ITE - IQ motif, EF-hand binding site & 488 \\
\hline IPR005123 & $\begin{array}{l}\text { Oxoglutarate/iron-dependent } \\
\text { dioxygenase }\end{array}$ & 484 \\
\hline IPR011527 & $\begin{array}{l}\text { ABC transporter type } 1 \text {, transmembrane } \\
\text { domain }\end{array}$ & 455 \\
\hline IPR013210 & $\begin{array}{l}\text { Leucine-rich repeat-containing } \\
\mathrm{N} \text {-terminal, plant-type }\end{array}$ & 444 \\
\hline IPR000008 & C2 domain & 442 \\
\hline IPR001623 & DnaJ domain & 428 \\
\hline IPR014001 & $\begin{array}{l}\text { Helicase superfamily } 1 / 2 \text {, ATP-binding } \\
\text { domain }\end{array}$ & 420 \\
\hline
\end{tabular}

\section{Gene ontology and KEGG pathway classification}

Gene ontology (GO) is an international classification system that provides standardized vocabulary for description of genes and their products functions in all organisms [58]. Here, to functionally categorize the pistachio transcripts, the best blast hits retrieved from the NR database was imported into Blast2GO software to capture GO terms and enzyme code numbers. Results specified that the maximum number of GO terms was achieved by
UniProtKB database and TAIR. Out of 84,117 sequences with NR annotation, a total of $68,539(80.76 \%)$ were associated with 302,375 GO terms (Fig. 5a), which classified into the three GO categories: biological process, molecular function, and cellular component. The majority of GO terms were assigned to the biological process $(13,7628,45.52 \%)$, followed by molecular function $(109,033$, $36.06 \%)$, and cellular component $(55,714,18.43 \%)$. We also surveyed the distribution of pistachio GO terms across the major sub-categories (third level) as illustrated in Fig. 5b. For the biological process category, genes involved in the "organic substance metabolic process", "primary metabolic process", and "cellular metabolic process" were highly represented. The most enriched GO terms in the molecular function category were "organic cyclic compound binding", "heterocyclic compound binding", and "ion binding". For the cellular component, transcripts with the most frequent GO terms being "cell”, "cell part", and "organelle". Accordingly, the annotated sequences involved in the main GO classification regulate the basic biological and metabolic processes, which were consistent with the most representative GO terms in the mango leaf and fruit transcriptome $[19,46]$. Since a portion of our transcriptome assembly was generated from plants under salt treatment, stress- and signaling-linked annotation were frequently found in this dataset. A total of 1753 sequences fell under 19 stressrelated GO terms, of which "response to salt stress" and "response to oxidative stress" were the main terms, constituting $37.2 \%$ and $21.5 \%$ of sequences, respectively. Similarly, 4009 sequences were distributed into 91 signaling pathwayrelated terms. The high frequency of GO terms associated with stress and signaling confirmed the sampling under salinity stress and properly making the transcriptome assembly. Of the 68,539 sequences associated with GO terms, a total of $21,598(31.5 \%)$ sequences were determined as enzyme. Among various enzyme types, transferases (37.35\%), followed by hydrolases (29.1\%) and oxidoreductases (20.62\%) were the most abundant enzymes (Fig. 5c). A large number of annotated enzymes in the present assembly proposes the presence of transcripts involved in various biological pathways $[59,60]$. Therefore, in order to fully recognize the active biological pathways in the $P$. vera transcriptome, the potential protein sequences were searched against the KEGG database using KAAS server with the single-directional best hit method and the corresponding KEGG orthology (KO) identifiers assigned to the sequences. KEGG is a highly integrated database providing information on biological systems and their relationships at the molecular, cellular and organism levels [61]. In total, 34,924 sequences were found to be involved in 279 KEGG pathways, covering five main KEGG categories, including metabolism, genetic information processing, organismal systems, cellular processes and environmental information processing (Fig. 6a) The 


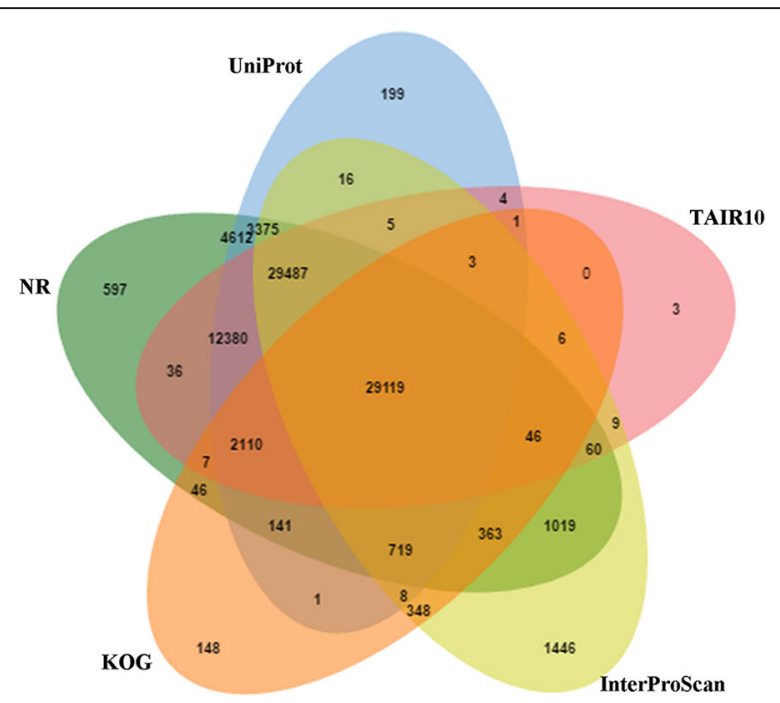

Fig. 4 Venn diagram shows the BLAST results of $P$. vera against five databases, including NR, UniProt, KOG, TAIR10, and InterProScan. The number of transcripts with significant hits is presented in each intersection of the Venn diagram

metabolic pathway (816 members), biosynthesis of secondary metabolites (397 members) and the biosynthesis of antibiotics (188 members) were the most representative pathways by the unique sequences. The top 10 pathways with the most assigned sequences were summarized in Fig. $6 \mathrm{~b}$ and the entire functional KEGG pathway classification of the $P$. vera transcriptome have been shown in Additional file 5. We believe that our findings will provide precious resources for investigating specific processes and functions in pistachio and other woody plants.

\section{Identification of transcription factors}

Under abiotic stresses, including salinity, many transcription factors (TF) operate to convert the stress-induced signals to plant cellular responses $[62,63]$. Over the past decades, numerous abiotic stress-related TFs have been determined in the various plants, but nothing has been studied in pistachio tree so far. As a result of homology search of $P$. vera transcriptome against the plant transcription factor database using BLASTX, a total of 21,099 transcripts were annotated and categorized into 57 families (Fig. 7). Of the various transcription factor families, $b H L H$, WRKY, MYB-related, and NAC were the four most abundant families with more than 1000 assigned transcripts while $N Z Z / S P$ and $L F Y$ had only one member (Additional file 6). Recent extensive genetic and molecular studies have proved that plenty of transcription factors belonging to the NAC, MYB, MYB-related, WRKY, bHLH, and $b Z I P$ families play central roles in plant responses to abiotic and biotic stresses [64-68]. Given that the activation of a large number of stress-responsive genes is mediated through specific TFs, identification of potential transcription factors will improve our understanding of the effective molecular mechanisms of abiotic stress tolerance in pistachio in future studies.

\section{Salt stress-related pathways and genes survey} Investigating the biological pathways play a key role in gaining insight into the advanced genomics researches. Plants response to abiotic stresses, including salinity is highly complex that involves various pathways, such as carbohydrate metabolism, biosynthesis of secondary metabolites, plant hormones biosynthesis and signal transduction pathways [69].

\section{Transcripts related to plant hormones}

Plant hormones trigger particular signaling cascades upon sensing the abiotic stress signals, which eventually results in an improved growth pattern under adverse conditions. The KEGG pathway mapping showed that the pistachio transcriptome assembly captures almost all key components of signal transduction pathways of all plant hormones, including auxin, cytokinin, gibberellin, abscisic acid (ABA), ethylene, brassinosteroid, jasmonic acid, and salicylic acid (Additional file 7). The prominent role of ABA during the plant salt response led us to examine ABA biosynthesis and its signal transduction pathway within the pistachio dataset. Through our KEGG analysis, we discover the main ABA biosynthesis pathway genes, including betacarotene 3-hydroxylase $(C R T z)$, beta-ring hydroxylase (LUT5), zeaxanthin epoxidase (ZEP), 9-cis-epoxycarotenoid dioxygenase $(N C E D)$ and ABA-aldehyde $(A B A 2)$ in the $P$. vera dataset (Additional file $8 \mathrm{a}$ ). Moreover, the enzymes of ABA 8'-hydroxylase (EC:1.14.13.93) required for $\mathrm{ABA}$ catabolism and ABA beta-glucosyltransferase (EC:2.4.1.263) involved in ABA inactivation by conjugation with glucose were also identified in our assembly. However, ABA function is required to hormone sense and transduce the signal to the cellular components. Here, we could detect all ABA signaling components such as the ABA receptor (PYR1), SnRK2 protein kinase, protein phosphatase of $P P 2 C$ and ABA responsive element binding factor $(A B F)$, which regulates the expression of ABA-responsive genes (Additional file $8 \mathrm{~b}$ ). Based on our results, most of the transcripts had multiple copies. For instance, the numbers of transcripts encoding ZEP, NCED, PYR1, and PP2C were $27,4,5$, and 33 , respectively.

Auxin is another multi-functional hormone that is responsible not only for normal plant growth and development, but also for modulating plant growth under stress conditions. Besides, it interacts with ABA, enhancing plant sensitivity to the ABA effect on root growth inhibition under osmotic stress [70]. Auxin biosynthesis initiates from the shikimate pathway, leading to the synthesis of aromatic amino acids, like Tryptophan 

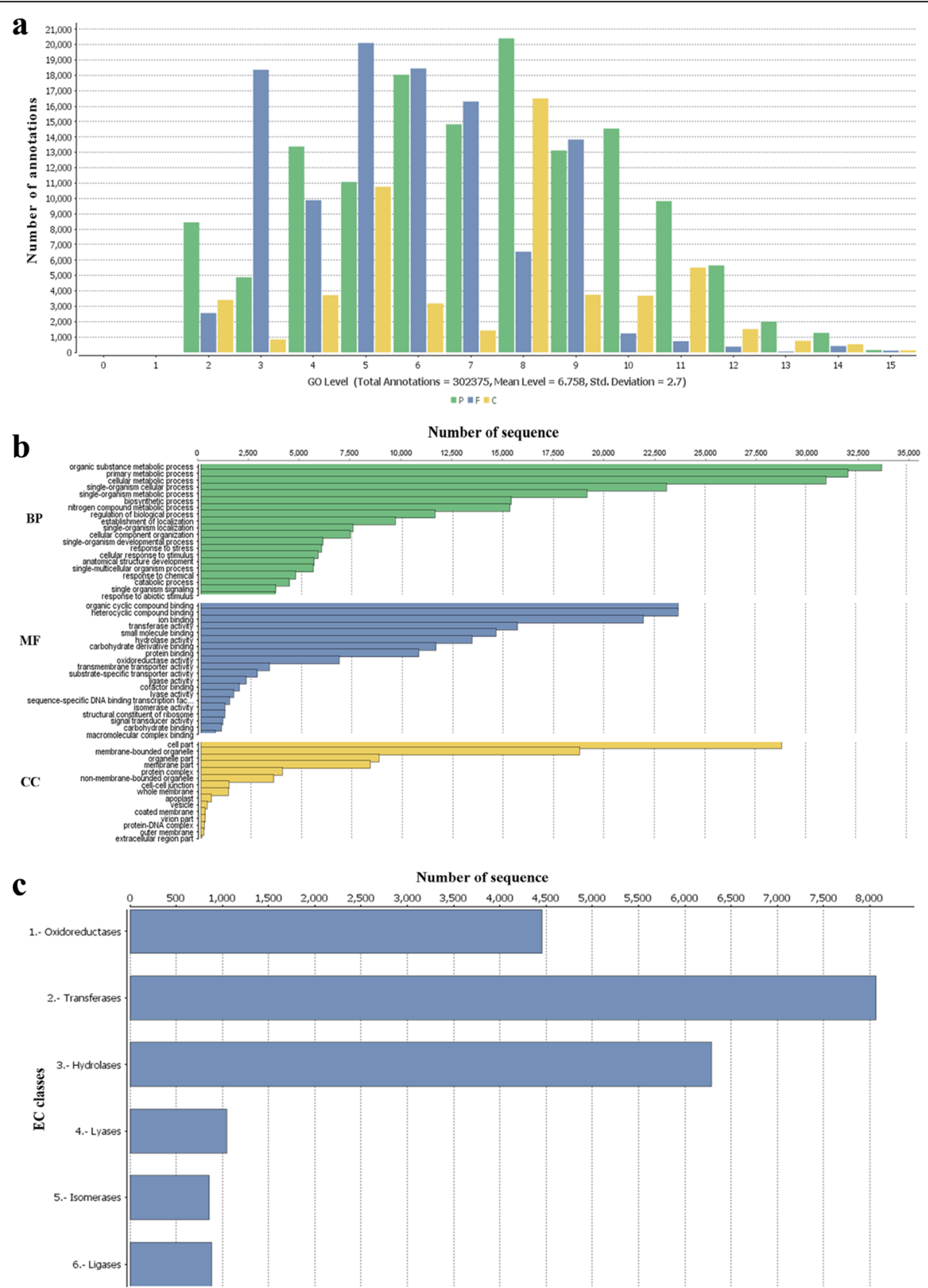

Fig. $5 \mathrm{GO}$ and enzyme classification of assembled pistachio transcripts. a GO level distribution of annotated sequences. b GO classification across three main categories. Biological process (BP), Molecular Function (MF), and Cellular Component (CC).c Catalytic activity distribution in annotated P. vera transcriptome

(Trp), a known precursor of auxin biosynthesis [71]. Additionally, the shikimate pathway is one of the crucial routes for primary and secondary metabolism in plants, which also generates precursors for the biosynthesis of different indole compounds, alkaloids, and other aromatic metabolites, lignin, as well as flavonoids. In our transcriptome dataset, all genes encoding for enzymes involved in the shikimate pathway were identified (Additional file 9a). Shikimate pathway-derived Trp converts into indole-3- acetic acid (IAA) through a two-step reaction that is catalyzed by aminotransferase of Arabidopsis (TAA) and YUCCA (YUC) family of flavin monooxygenases [71]. This auxin biosynthesis pathway is a major route for auxin production, which is conserved among plants, including pistachio as our data analysis revealed the corresponding transcripts. Furthermore, the $P$. vera transcriptome dataset contained enzymes like indoleacetaldoxime dehydratase (EC: 4.99.1.6), cytochrome P450 monooxygenase 

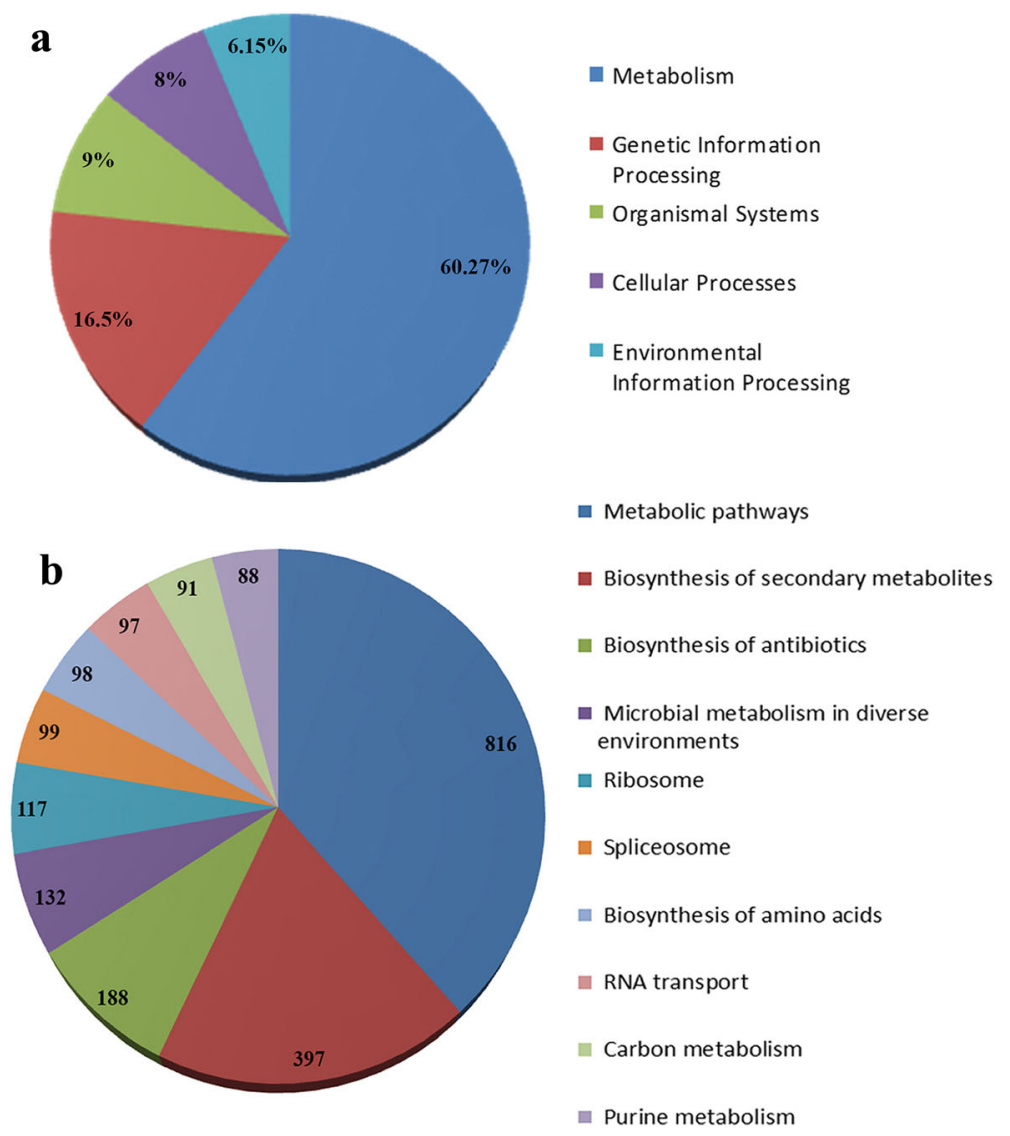

Fig. 6 KEGG classification of pistachio transcriptome. a KEGG distribution of annotated transcripts into biological categories. b The top 10 pathways with the highest transcript numbers

(EC:1.14.-.), amidase (EC:3.5.1.4), and indole-3acetaldehyde oxidase (EC:1.2.3.7) that contributed to the auxin biosynthesis from alternative pathways (Additional file 9b). In addition to these key biosynthetic genes, all genes encoding proteins in the auxin

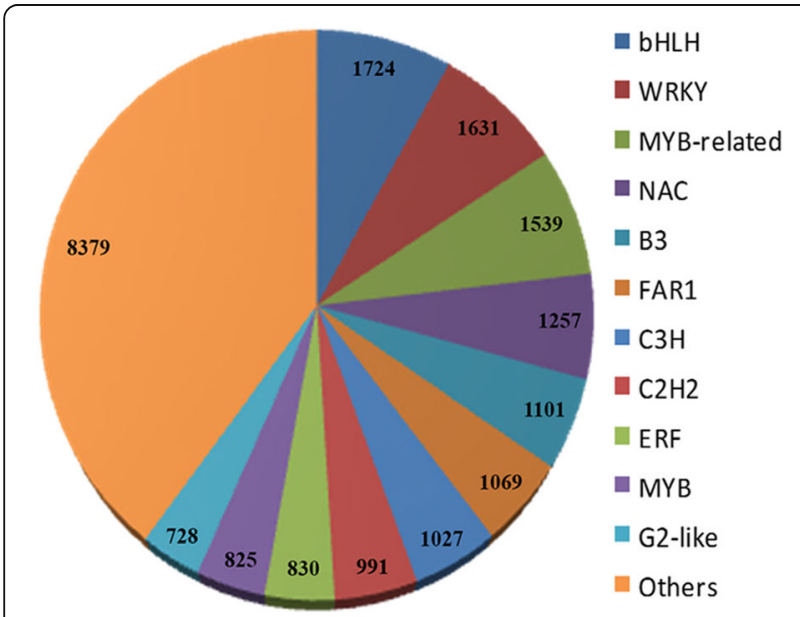

Fig. 7 Distribution of $P$. vera transcripts into different transcription factor families signal transduction pathway, auxin influx carrier (AUX1), transport inhibitor response1 (TIR1), Aux/IAA repressor protein, $A R F$ transcription factor, $G H 3$, and SAUR were found in the pistachio transcriptome dataset (Additional file 9c). Considering the major role of $\mathrm{ABA}$ in plant abiotic stresses response and its interaction with auxin, our findings offer valuable information to decipher the ABAregulated processes and discover the probable stress response mechanisms in pistachio.

\section{Transcripts related to flavonoid biosynthesis}

Flavonoids constitute a major group of polyphenolic secondary metabolites with a vast array of biological functions in plants, including stress protection. They operate as an antioxidant defense system for protecting plants against oxidative damage originating from various abiotic and biotic stresses $[72,73]$. The biosynthesis of flavonoids requires the enzymes involved in the phenylpropanoids pathway and its flavonoid branch pathways. In the present study, KEGG analysis of the $P$. vera transcriptome showed the presence of 18 transcripts to be contributed to the biosynthesis of different compounds of phenylpropanoid pathway (Additional file 10). In our dataset, starting from 
initial enzymes of flavonoids biosynthesis (through the phenylpropanoid pathway), such as phenylalanine ammonia lyase (PAL) (EC: 4.3.1.24), cinnamate 4- monooxygenase (EC: 1.14.13.11), 4-coumarate CoA ligase (EC: 6.2.1.12), and chalcone synthase (EC: 2.3.1.74) were recognized. PAL, the key enzyme in the phenolic biosynthesis pathway, is generally considered as a biochemical marker of stress conditions. It has been reported that the PAL enzymatic activity is different across various $P$. vera cultivars and there is a positive correlation among the PAL activity, phenolic compounds and the higher tolerance of pistachio cultivar against environmental stresses [74]. Here, ten transcripts encoding $P A L$ were identified in the pistachio transcriptome that could be potentially used as molecular markers for selecting the salt-tolerant pistachio species. Similarly, it has been recently proved that the chalcone synthase, at both transcript and protein levels, was positively related to salinity tolerance in the salttolerant soybean genotypes [75], indicating the pivotal role of flavonoids biosynthesis in salinity tolerance. Further, chalcone isomerase (EC: 5.5.1.6) that catalyzes chalcone isomerisation into naringenin was identified in this dataset. Naringenin is a central branch point for the synthesis of several major groups of flavonoids, including flavanones, flavonols, and anthocyanins [76]. Interestingly, all transcripts encoding the enzymes participated in the biosynthesis of the main class of flavonoids were discovered in the present transcriptome (Additional file 11). We proposed the biosynthesis pathway for major flavonoids in pistachio in Fig. 8. Briefly, flavonoids are synthesized via the phenylpropanoid pathway where phenylalanine is converted to coumaroyl-CoA through a series of enzymatic modifications. Coumaroyl-CoA is then combined with malonyl-CoA to yield chalcone, which catalyzed by chalcone synthase, and isomerized into naringenin via chalcone isomerase activity. The metabolic pathway continues through a series of enzymatic reactions to yield flavanones, dihydroflavonols, and anthocyanins. Flavonoids of quercetin, luteolin, myricetin, and kaempferol have been detected in the leaf extracts of pistachio through highperformance liquid chromatography (HPLC) analysis. In the case of anthocyanins, only two types of cyanidin

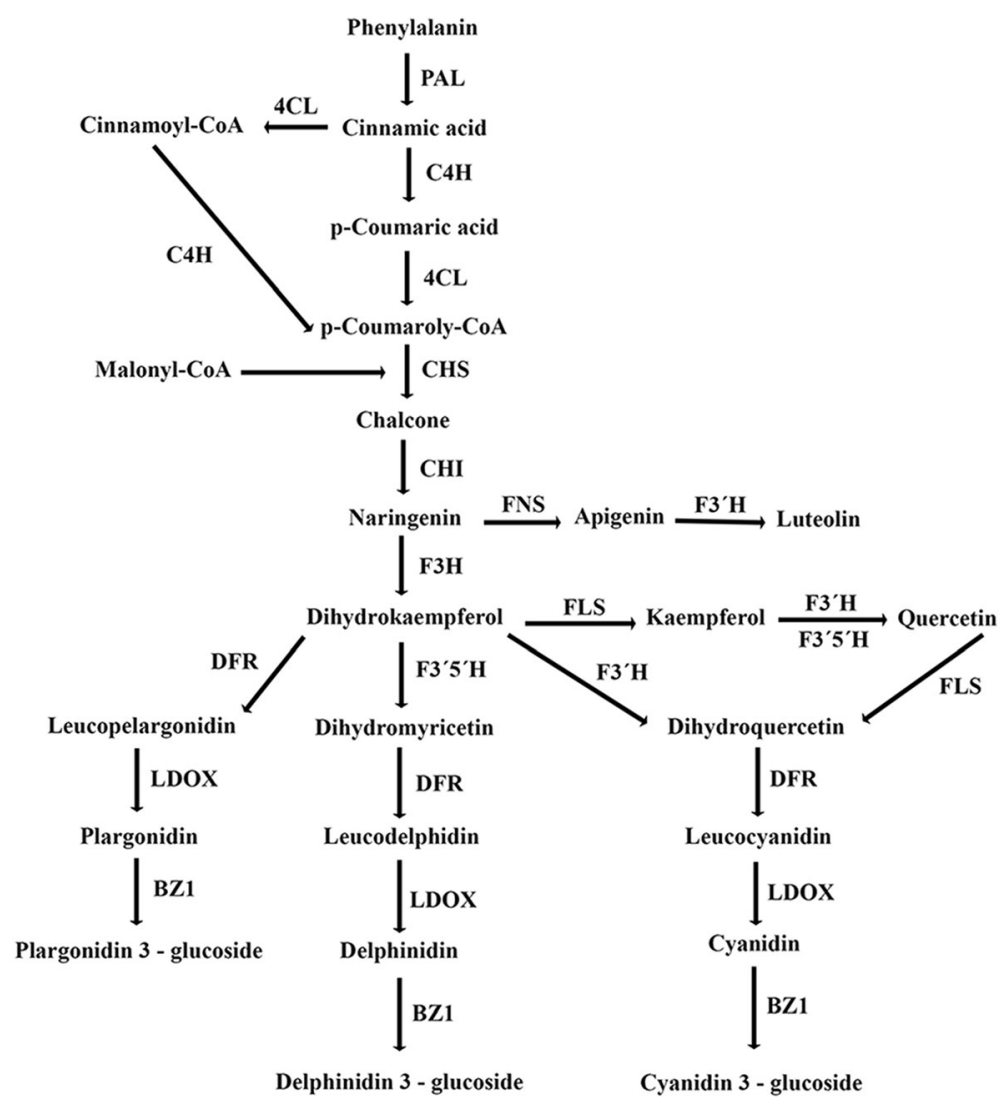

Fig. 8 The proposed pathway for the biosynthesis of major flavonoids, including luteolin, quercetin, kaempferol and various types of anthocyanins (pelargonidin, delphinidin, and cyanidin) in pistachio. Phenylalanine ammonia-lyase (PAL), 4-coumarate CoA ligase (4CL), Trans-cinnamate 4-monooxygenase (C4H), Chalcone synthase (CHS), Chalcone isomerase (CH), Flavone synthase (FNS), Flavonoid-3'-hydroxylase (F3'H), Flavone-3hydroxylase (F3H), Flavonol synthase (FLS), Flavonoid 3'5'-hydroxylase (F3'5'H), Dihydroflavonol-4-reductase (DFR), Leucoanthocyanidin dioxygenase (LDOX), Anthocyanidin 3-O-glucosyltransferase (BZ1). The genes listed in this Fig. were all identified in $P$. vera transcriptome 
(cyanidin 3-galactoside and cyanidin 3-glucoside) were experimentally reported in the pistachio nut [77]. However, detecting the active biological pathways related to other anthocyanin types may imply the presence of corresponding anthocyanins, including delphinidin and pelargonidin in pistachio (Fig. 8).

\section{Transcripts related to stress-responsive genes}

Salt stress tolerance is considered a quantitative trait, requiring the contribution of several genes for plant survival under adverse conditions. While a large number of stress-responsive genes were determined in various plant species, there is no report for pistachio that can prevent the understanding of stress tolerance mechanism in this major salt-tolerant tree. Due to similar signal transduction pathways and plant responses among abiotic stresses, like salinity, drought and cold, a BLASTX analysis (e-value cutoff of 1e-5) against a database made from abovementioned stresses-responsive genes (see Methods) were conducted and 13,097 transcripts as candidate stressassociated genes identified. The current pistachio transcriptome assembly represented 617 out of the 704 abiotic stress-related proteins, suggesting our assembly enough enriched with genes involved in the various aspects of salt, drought and cold stresses.

The products of stress-inducible genes can be classified into two major groups; the first group is comprised of functional proteins, like late embryogenesis abundant (LEA) proteins, osmotin and chaperones and the second group contains the regulatory proteins, such as transcription factors, protein kinases and protein phosphatases that involved in the regulation of stress-responsive genes expression and signaling pathways [78]. Here, 116 LEA, 9 osmotin, and 160 chaperone transcripts were recognized in the pistachio transcriptome assembly. The expression of a large number of stress-responsive genes is regulated by the mitogen-activated protein kinase (MAPK) cascade. The MAPK signaling pathway consists of three components, MAPK kinase kinase (MAPKKK), MAPK kinase (MAPKK) and MAPK that play a pivotal role in transferring the extracellular signals to the nucleus [79]. In the current transcriptome assembly, 332 transcripts encoding the various members of MAPK pathway responding to abiotic stresses were identified. Among them, MAPK4 and MAPK6 with 230 transcripts were the most abundant transcripts, which is associated with their function during salt stress. The both MAPK4 and MAPK6 are positively involved in the plant abiotic stresses (salt, drought, and cold); Arabidopsis overexpressors MAPK4 and MAPK6, constitutively expressed stress-induced genes, resulting in an increased salinity and freezing tolerance [80]. One of the downstream targets of MAPK6 is a plasma membrane $\mathrm{Na}^{+} / \mathrm{H}^{+}$antiporter (SOS1) [81], which mediates $\mathrm{Na}^{+}$efflux from the roots and loading of $\mathrm{Na}^{+}$ions in the xylem. The salt overly sensitive (SOS) pathway that comprises SOS1, SOS2, and SOS3 has emerged as a main mechanism to maintain ion homeostasis in plants under salt stress. Salinity evokes a cytosolic calcium signal that is perceived by SOS3 and activates SOS2, a serine/threonine protein kinase. The SOS2-SOS3 complex regulates the expression level and activity of SOS1 [82]. Our pistachio transcriptome assembly represented all components of this pathway, so that 11, 7, and 16 transcripts encoding SOS1, SOS2, and SOS3, respectively, were recognized. Therefore, the SOS pathway is conserved in pistachio tree and presumably conferred salt tolerance in salt-tolerant pistachio species. In the case of salt stress signaling, in addition to SOS3 family, calcium-dependent protein kinase (CDPK) and calcineurin B-like protein-interacting protein kinase (CIPK) have emerged to be the main proteins in linking of stress signal to specific protein phosphorylation cascades [83]. We also characterized 94 stress-responsive transcripts corresponding to calcium-dependent protein kinase $(C D P K)$ and 133 transcripts encoding calcineurin B-like protein-interacting protein kinase $(C I P K)$ in the present assembly.

One of the common consequences of abiotic stresses is the increased reactive oxygen species (ROS) content [84]. Although ROS are considered as the signaling molecules with the regulatory role during plant development and abiotic stress responses, plants have evolved enzymatic and non-enzymatic antioxidants to maintain the steadystate level of ROS and alleviate the oxidative stress [85]. Considering the central role of enzymatic antioxidants, the assembled transcriptome was surveyed for stressresponsive transcripts encoding this type of enzymes. As a result, 24 transcripts for superoxide-dismutase (SOD), 88 glutathione S-transferase and 204 peroxidase family transcripts were identified. In the case of $S O D$, $\mathrm{Cu}-\mathrm{Zn} S O D$ was the main isoform that correlated with its broad localization in cytosol, apoplast, peroxisomes and chloroplasts.

\section{Gene validation and expression analysis}

In order to verify that the reconstructed transcripts were expressed, several key stress-responsive genes, including $Z E P$ and NCED3 contributing to ABA biosynthesis along with $P P 2 C A$ to be involved in the ABA signaling pathway, as well as $N H X 7$, sodium/proton antiporter known as SOS1, dehydrin, a member of group 2 LEA and $C D P K 11$ were selected to perform qRT-PCR analysis in root samples of $P$. vera L. cv. Ghazvini and Sarakhs after $0,6,24$, and $48 \mathrm{~h}$ of salt treatment. All primers pair generated a single band with the expected size on the gene electrophoresis and one peak on the melting curve, implying that the assembled transcripts were reliable and subsequent gene expression profiling were conceivable. Based on our results, the expression of ZEP gene was 
up-regulated up to $48 \mathrm{~h}$ of salt treatment in the salt-tolerant cultivar (Ghazvini) while the slightly up-regulation by 1.25 fold until $24 \mathrm{~h}$ and the significant down-regulation by -2.1 fold after $48 \mathrm{~h}$ of salinity were observed in the salt-sensitive cultivar (Sarakhs) (Fig. 9a).

The transcript level of NCED3 was found to be higher in the salt-tolerant than salt-sensitive cultivar. As shown in Fig. 9b, the abundance of NCED3 transcript was increased at the 6 and $24 \mathrm{~h}$ and slightly decreased (fold change of -0.98) after $48 \mathrm{~h}$ exposure to stress in the salt-tolerant cultivar. But, in the salt-sensitive cultivar, NCED3 expression level was enhanced only over the $6 \mathrm{~h}$ of the treatment, then considerably reduction by -9 fold occurred at the $48 \mathrm{~h}$ of salinity.

ZEP and NCED are the key enzymes in the ABA biosynthesis pathway that contribute to convert the zeaxanthin to violaxanthin and epoxycarotenoids to xanthoxin, respectively [86]. In agreement with previous studies that revealed the elevated expression of ZEP and NCED3 under salt stress in Arabiopsis thaliana [83] and Populus tomentosa [87], we found the similar trend, especially for salt-tolerant cultivar in the present study. Considering the positive correlation between the increased level of these transcripts and ABA content, higher salinity tolerance of Ghazvini as compared to Sarakhs can probably result from the more ABA production under salt treatment. In accordance with these results, the more ABA accumulation in $P$. vera L. cv. Badami (another salttolerant cultivar) than $P$. vera $\mathrm{L}$. cv. Sarakhs under salt treatment has been reported by Panahi (2009) [88]. The more ABA content in the salt-tolerant maize [89] and tomato [90] under salinity has been previously demonstrated. The expression analysis of $P P 2 C$, one of the main genes in ABA signaling pathway revealed that its transcript level was enhanced in both cultivars, Ghazvini and Sarakhs, during salinity stress. As shown in Fig. 9c, this up-regulation is slightly but significantly higher in Ghazvini than Sarakhs. The protein phosphatase of PP2CA, as a negative regulator, mediates plant responses to salt stress by regulation of $\mathrm{ABA}$ signaling pathway. PP2CA gene is highly induced by salinity and ABA as shown by previous researches in Oryza sativa [91] and Chrysanthemum [92]. From the PP2C expression pattern, like other plants, a transcriptional negative feedback mechanism may regulate the $\mathrm{ABA}$ response in the both pistachio cultivars under salinity treatment.

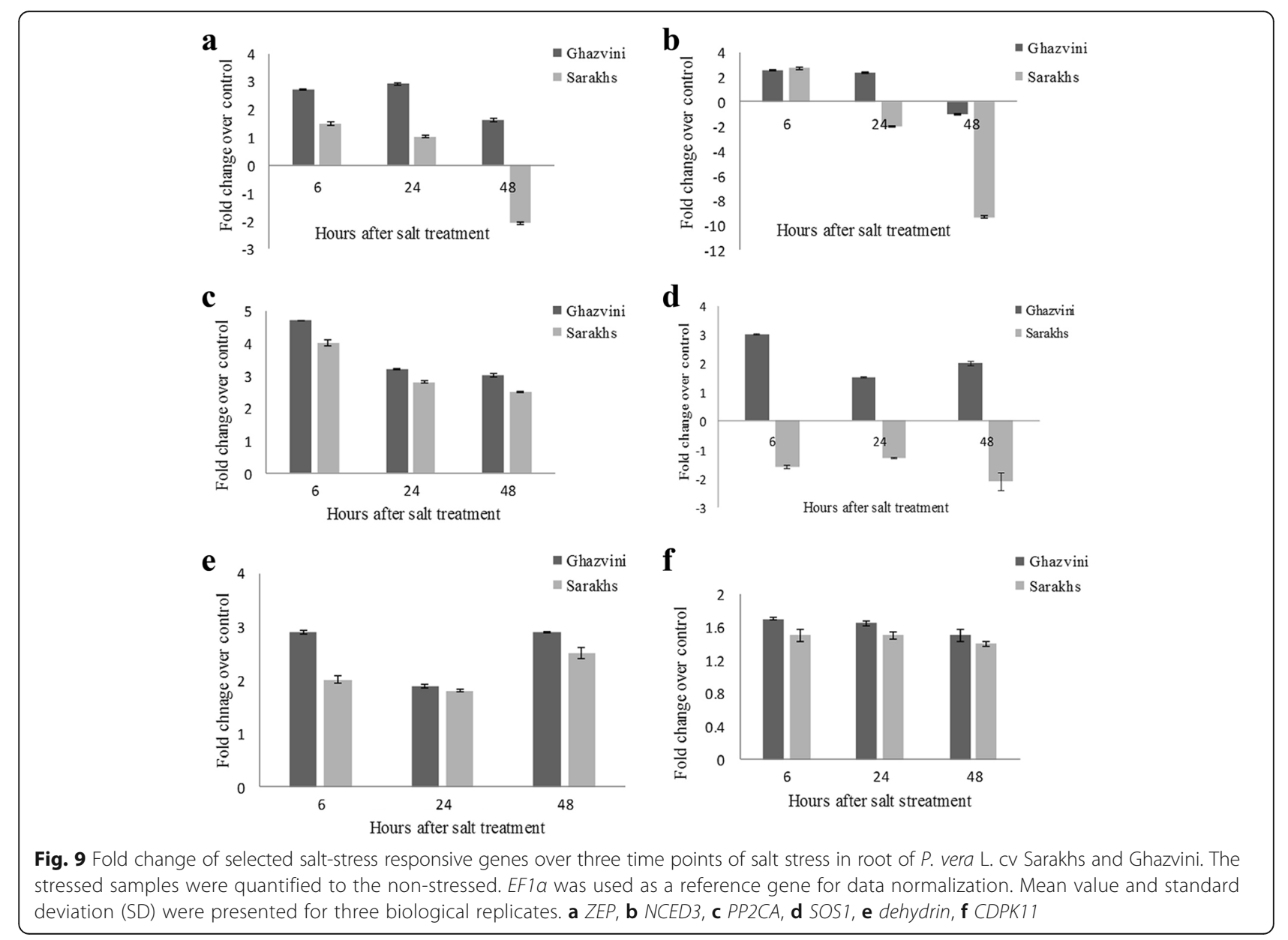


SOS1, one of the central components of SOS pathway, exhibited the contrasted expression pattern between salttolerant and salt-sensitive pistachio cultivars (Fig. 9d). The transcript level of SOS1 was significantly reduced over all three time points in Sarakhs, whereas its abundance was increased after 24 and 48 h of salinity in Ghazvini. Interestingly, when compared with Sarakhs, we detected a lower accumulation of $\mathrm{Na}^{+}$in roots of Ghazvini (unpublished data), which may correlate with the upregulation of SOS1 in the salt-tolerant cultivar. Therefore, pistachio salinity tolerance may partly refer to the active SOS pathway and sodium extrusion from cytoplasm. The similar result has been previously reported in salt-tolerant cotton genotype, Gossypium hirsutum [93].

As Fig. 9e and $\mathrm{f}$ show, in the both cultivars, expression of dehydrin and CDPK11 were induced at all time points of salt stress. However, the genes up-regulation is slightly higher in salt-tolerant cultivar than salt-sensitive cultivar. In consistent with our results, the up-regulation of both genes in response to drought and high salinity stresses has been proved [93, 94]. Overall, the expression analysis confirmed that the selected stress-responsive genes are expressed at higher level in tolerant cultivar than sensitive one in response to salt treatment. However, further gene expression analysis at the large scale is required to exactly decipher the pistachio salt tolerance mechanism.

\section{Characterization of simple sequence repeats (SSRs)}

SSRs are one of the most informative and versatile molecular markers, which are commonly used in genetic diversity evaluation, conservation genomics and genetic mapping studies [95]. In order to identify and conserve the salt-tolerant pistachio rootstocks, which is classified as a near threatened by IUCN (International Union for Conservation of Nature), the development of SSR markers is highly desirable. Therefore, we screened all contigs resulted from Trinity to discover potential SSRs for future researches. In total, 11,337 SSRs defined as di-to hexanucleotide motifs were recognized in 11,130 contigs, with 204 contigs bearing more than one SSR. Di- and trinucleotide repeats were the most abundant SSRs, accounting for $40-44 \%$ of total SSRs, followed by tetra- (9.5\%), panta(3.1\%) and hexanucleotide repeats (2.2\%) (Table 5), which is consistent with other plants [96-98]. The pistachio transcriptome was rich in GA/TC (12.13\%), AG/CT (11.02\%), AT/AT (8.32\%), TA/TA (8.04\%), GAA/TTC (5\%) and AGA/TCT (4.02\%). To make these SSR markers useful, Primer 3 was applied to design primer pairs for each SSR. A total of 7605 primer pairs were generated from the microsatellites with sufficient flanking sequences. All primer pairs were submitted to virtual PCR within SSR Locator tool that 2702 of them produced a single amplicon, suggesting the specificity of the corresponding
Table 5 Statistics of SSRs in the P.vera transcriptome

\begin{tabular}{ll}
\hline Total number of sequences & 144,103 \\
Total number of SSRs & 11,337 \\
Number of SSR containing sequences & 11,130 \\
Number of dinucleotide repeats & $5063(44.7 \%)$ \\
Number of trinucleotide repeats & $4605(40.6 \%)$ \\
Number of tetranucleotide repeats & $1075(9.5 \%)$ \\
Number of pentanucleotide repeats & $350(3.1 \%)$ \\
Number of hexanucleotide repeats & $244(2.2 \%)$ \\
\hline
\end{tabular}

SSR marker (Additional file 12). It has been reported that SSR markers that generate one in silico PCR product should be the putative single-locus markers and could be especially useful [98]. This large-scale marker discovery facilitates future researches for finding the salt tolerance-related markers and conserving the tolerant pistachio cultivars.

\section{Conclusion}

The present study is the first attempt at investigating the whole transcriptome sequencing of $P$. vera. A total of 368,953,262 million clean PE reads were created from various tissues of two pistachio cultivars with contrasting salinity tolerance under the control and salinity conditions by Illumina Hiseq 2000 platform. Following the comprehensive comparison of different assemblers using multiple length- and annotation-based metrics, the Trinity assembly was selected and functionally annotated against several protein databases. A total of 29,119 sequences received the best hit with known homologous proteins in all five used protein databases. The highest number of transcripts has been assigned to the NAC, MYB, MYB-related, WRKY, bHLH, and bZIP transcription factors families, consistent with their critical roles in plant salt stress responses. Most genes involved in plant hormone biosynthesis and signaling pathways as well as pathways to be contributed to secondary metabolite biosynthesis were found in pistachio transcriptome assembly. We also propose the biosynthetic pathway of major flavonoids in pistachio. A total of 13,097 transcripts found to be the stress-responsive and some of them validated by qRT-PCR, further confirm the accuracy of assembly. Further, 11,337 SSRs with a frequency of $14.4 \mathrm{~kb}$ were identified, which the most abundant being dinucleotide repeats. Taken together, given the usage of various pistachio tissues (leaf, stem, and root) and the similarity in signaling pathways and plant responses during salt, drought, and cold stresses, the current research could provide a valuable foundation for future RNA-seq analysis of $P$. vera under any of these stresses to discover the potential molecular mechanisms of abiotic stresses response and accelerate the breeding of new cultivars with more tolerance against abiotic stresses. 


\section{Additional files}

Additional file 1: Plant survival rate, $\mathrm{Na}^{+}$and $\mathrm{K}^{+}$level and Malondialdehyde (MDA) concentration parameters used for selecting the salt-sensitive and salt-tolerant cultivars. (DOCX $220 \mathrm{~kb}$ )

Additional file 2: Primer sequence used for qRT-PCR analysis. (DOCX $15 \mathrm{~kb}$ )

Additional file 3: Top BLAST hits from NCBI NR database with corresponding GO terms, Enzyme code and InterPro IDs. (XLSX 5822 kb)

Additional file 4: Eukaryotic orthologous groups (KOG) functional classification of pistachio transcriptome. A) RNA processing and modification; B) Chromatin structure and dynamics; C) Energy production and conversion; D) Cell cycle control, cell division, chromosome partitioning; E) Amino acid transport and metabolism; F) Nucleotide transport and metabolism; G) Carbohydrate transport and metabolism; H) Coenzyme transport and metabolism; I) Lipid transport and metabolism; J) Translation, ribosomal structure and biogenesis; K) Transcription; L) Replication, recombination and repair; M) Cell wall/membrane/envelope biogenesis; N) Cell motility; O) Post-translational modification, protein turnover, chaperones; P) Inorganic ion transport and metabolism; Q) Secondary metabolites biosynthesis, transport and catabolism; R) General function prediction only; S) Function unknown; T) Signal transduction mechanisms; U) Intracellular trafficking, secretion, and vesicular transport; V) Defense mechanisms; W) Extracellular structures; Y) Nuclear structure; Z) Cytoskeleton. (JPEG $84 \mathrm{~kb})$

Additional file 5: KEGG pathway classification of $P$. vera transcriptome (XLSX $19 \mathrm{~kb}$ )

Additional file 6: The reconstructed pistachio sequences that shows significant homology with the transcription factors (XLSX $10 \mathrm{~kb}$ )

Additional file 7: Plant hormone signal transduction pathway. The ortholog genes in pistachio indicated by green color. (JPEG 618 kb)

Additional file 8: Genes involved in the carotenoid biosynthesis in the pistachio transcriptome as shown by pink highlighting. a) ABA biosynthesis pathway. b) ABA signal transduction pathway. (JPEG 1096 kb)

Additional file 9: Genes involved in the shikimate biosynthesis a), auxin biosynthesis b), and auxin signal transduction pathways c) within pistachio transcriptome, as indicated by pink color. (JPEG 1494 kb)

Additional file 10: Phenylpropanoid biosynthesis pathway. The ortholog genes in pistachio transcriptome indicated by green highlighting. (JPEG 704 kb)

Additional file 11: Flavonoid biosynthesis pathway. The ortholog genes in pistachio transcriptome specified by green highlighting. (JPEG 608 kb)

Additional file 12: Primer pairs was used for virtual PCR to amplify SSR repeats. (XLSX $395 \mathrm{~kb}$ )

\section{Abbreviations}

ABA: Abscisic acid; CEGMA: Core eukaryotic genes mapping approach; CTAB: Cetyltrimethyl ammonium bromide; DETONATE: De novo transcriptome rnaseq assembly with or without the truth evaluation; EF1a: Elongation factor 1 alpha; EST: Expressed sequence tag; GO: Gene ontology; HPLC: High-performance liquid chromatography; KEGG: Kyoto encyclopedia of genes and genomes; KO: KEGG orthology; KOG: Eukaryotic orthologous groups; LEA: Late embryogenesis abundant; MAPK: Mitogen-activated protein kinase; MDA: Malondialdehyde; NCBI: National center for biotechnology information; OHR: Ortholog hit ratio; PE: Paired-end; PPR: Pentatricopeptide repeat; qRT-PCR: Quantitative real-time PCR; RAPD: Random amplified polymorphic DNA; RBH: Reciprocal best hits; RIN: RNA integrity number; RMBT: Reads mapped back to assembled transcripts; RNA-seq: RNA sequencing: $\mathrm{SBH}$ : Single-directional best hit; SOS: Salt overly sensitive; SSR: Simple sequence repeats; TAIR: Arabidopsis information resource; TF: Transcription factors; Trp: Tryptophan

\section{Acknowledgements}

The authors appreciate the partial financial support for this work by the Iran National Science Foundation (INSF), Tehran, Iran.

\section{Funding}

This research was partially supported by Iran National Science Foundation (INSF).

\section{Availability of data and materials}

Raw sequencing reads produced in this study have been deposited at NCBI in the Short Read Archive (SRA) database under the accession number SRX1880621.

\section{Authors' contributions}

MMZ and SMS designed the research. MMZ carried out the experiment, analyzed the data and drafted the manuscript. SMS, EE, ME, GDM, and CB supervised and assisted in the NGS data analysis and prepared the final manuscript. All authors read and approved the manuscript.

\section{Ethics approval and consent to participate}

Not applicable.

\section{Consent for publication}

Not applicable.

\section{Competing interests}

The authors declare that they have no competing interests.

\section{Publisher's Note}

Springer Nature remains neutral with regard to jurisdictional claims in published maps and institutional affiliations.

\section{Author details}

${ }^{1}$ Plant Biotechnology Department, National Institute of Genetic Engineering and Biotechnology, Tehran, Iran. ${ }^{2}$ School of Medicine, The University of Adelaide, Adelaide, Australia. ${ }^{3}$ Institute of Biotechnology, Shiraz University, Shiraz, Iran. ${ }^{4}$ Division of Information Technology, Engineering and the Environment, School of Information Technology and Mathematical Sciences, University of South Australia, Adelaide, Australia. ${ }^{5}$ School of Biological Sciences, Faculty of Science and Engineering, Flinders University, Adelaide, Australia. ${ }^{6}$ Department of Biology, University of Qom, Qom, Iran. 'Center of Marine Sciences (CCMAR), University of Algarve, Faro, Portugal. ${ }^{8}$ Department of Biological Sciences, Chicago State University, Chicago, IL, USA.

Received: 7 February 2017 Accepted: 1 August 2017

Published online: 17 August 2017

\section{References}

1. Zohary M. A monographical study of the genus Pistacia. Palestine J Bot (Jerusalem Ser). 1952:5(4):187-228.

2. Crane JC. Pistachio tree nuts. Westport: Avipublishing Company; 1978

3. F Faostat. 2016. FAO web page. http://www.fao.org/faostat. Accessed 14 Dec 2016.

4. Bozorgi M, Memariani Z, Mobli M, Salehi Surmaghi MH, Shams-Ardekani MR Rahimi R. Five Pistacia species (P. vera, P. atlantica, P. terebinthus, P. khinjuk, and $P$. lentiscus): a review of their traditional uses, phytochemistry, and pharmacology. ScientificWorldJournal. 2013;15:1-33.

5. Tsokou A, Georgopoulou K, Melliou E, Magiatis P, Tsitsa E. Composition and enantiomeric analysis of the essential oil of the fruits and the leaves of Pistacia vera from Greece. Molecules. 2007;12(6):1233-9.

6. Ferguson L, Poss J, Grattan S, Grieve C, Wang D, Wilson C, Donovan T, Chao C-T. Pistachio rootstocks influence scion growth and ion relations under salinity and boron stress. J Am Soc Hortic Sci. 2002:127(2):194-9.

7. Sofo A, Palese AM, Casacchia T, Dichio B, Xiloyannis C. Sustainable fruit production in Mediterranean orchards subjected to drought stress. In: Ahmad P., Prasad M. (eds) Abiotic Stress Responses in Plants. New York, NY: Springer; 2012. p. 105-129.

8. Hajiboland R, Norouzi F, Poschenrieder C. Growth, physiological, biochemical and ionic responses of pistachio seedlings to mild and high salinity. Trees. 2014:28(4):1065-78

9. Jazi MM, Khorzoghi EG, Botanga C, Seyedi SM. Identification of reference genes for quantitative gene expression studies in a non-model tree pistachio (Pistacia vera L.). PLoS One. 2016;11(6):e0157467.

10. Motalebipour EZ, Kafkas S, Khodaeiaminjan M, Coban N, Gözel H. Genome survey of pistachio (Pistacia vera L.) by next generation sequencing: development of novel SSR markers and genetic diversity in Pistacia species. BMC Genomics. 2016;17(1):998 
11. Vendramin E, Dettori M, Verde I, Micali S, Giovinazzi J, Mardi M, Avanzato D, Quarta R. Molecular characterization of Pistacia genus by microsatellite markers. Acta Hortic. 2009;825:55-61.

12. Ahmad R, Ferguson L, Southwick SM. Identification of pistachio (Pistacia vera L. ) nuts with microsatellite markers. J Am Soc Hortic Sci. 2003;128(6):898-903.

13. Zaloğlu S, Kafkas S, Doğan Y, Güney M. Development and characterization of SSR markers from pistachio (Pistacia vera L.) and their transferability to eight Pistacia species. Sci. Horticulture. 2015;189:94-103.

14. Kamiab F, Ebadi A, Panahi B, Tajabadi A. RAPD analysis for sex determination in Pistacia vera L. J Nuts. 2014;5:51-5.

15. Sepaskhah A, Maftoun M. Relative salt tolerance of pistachio cultivars. J Hortic Sci. 1988;63(1):157-62.

16. Abbaspour H, Saeidi-Sar S, Afshari H, Abdel-Wahhab M. Tolerance of mycorrhiza infected pistachio (Pistacia vera L.) seedling to drought stress under glasshouse conditions. J Plant Physiol. 2012;169(7):704-9.

17. Chelli-Chaabouni A, Mosbah AB, Maalej M, Gargouri K, Gargouri-Bouzid R, Drira N. In vitro salinity tolerance of two pistachio rootstocks: Pistacia vera $\mathrm{L}$. and P. atlantica Desf. Environ Exp Bot. 2010;69(3):302-12.

18. Lotfi A, Jahanbakhshian Z, Faghihi F, Seyedi SM. The effect of salinity stress on survival percentage and physiological characteristics in three varieties of pistachio (Pistacia vera). Biologia. 2015;70(9):1185-92.

19. Dautt-Castro M, Ochoa-Leyva A, Contreras-Vergara CA, Pacheco-Sanchez MA, Casas-Flores S, Sanchez-Flores A, Kuhn DN, Islas-Osuna MA. Mango (Mangifera indica L.) cv. Kent fruit mesocarp de novo transcriptome assembly identifies gene families important for ripening. Front Plant Sci. 2015;6:62.

20. Dong S, Liu Y, Xiong B, Jiang X, Zhang Z. Transcriptomic analysis of a potential bioenergy tree, Pistacia chinensis Bunge, and identification of candidate genes involved in the biosynthesis of oil. Bioenergy Res. 2016;9(3):740-9.

21. Chopra R, Burow G, Farmer A, Mudge J, Simpson CE, Burow MD. Comparisons of de novo transcriptome assemblers in diploid and polyploid species using peanut (Arachis spp.) RNA-seq data. PLoS One. 2014:9(12):e115055.

22. Duan J, Xia C, Zhao G, Jia J, Kong X. Optimizing de novo common wheat transcriptome assembly using short-read RNA-Seq data. BMC Genomics. 2012;13(1):1

23. Pont C, Murat F, Confolent C, Balzerque S, Salse J. RNA-seq in grain unveils fate of neo-and paleopolyploidization events in bread wheat (Triticum aestivum L.). Genome Biol. 2011;12(12):1-19.

24. O'Neil ST, Emrich SJ. Assessing de novo transcriptome assembly metrics for consistency and utility. BMC Genomics. 2013;14(1):1.

25. Jazi MM, Rajaei S, Seyedi SM. Isolation of high quality RNA from pistachio (Pistacia vera L.) and other woody plants high in secondary metabolites. Physiol Mol Biol Plants. 2015;21(4):597-603.

26. Kopylova E, Noé L, Touzet H. SortMeRNA: fast and accurate filtering of ribosomal RNAs in metatranscriptomic data. Bioinformatics. 2012;28(24):3211-7.

27. Haas BJ, Papanicolaou A, Yassour M, Grabherr M, Blood PD, Bowden J, Couger MB, Eccles D, Li B, Lieber M. De novo transcript sequence reconstruction from RNA-seq using the trinity platform for reference generation and analysis. Nat Protoc. 2013;8(8):1494-512.

28. Xie Y, Wu G, Tang J, Luo R, Patterson J, Liu S, Huang W, He G, Gu S, Li S. SOAPdenovo-trans: de novo transcriptome assembly with short RNA-Seq reads. Bioinformatics. 2014;30(12):1660-6.

29. He B, Zhao S, Chen Y, Cao Q, Wei C, Cheng X, Zhang Y. Optimal assembly strategies of transcriptome related to ploidies of eukaryotic organisms. BMC Genomics. 2015;16(1):1.

30. Huang X, Madan A. CAP3: a DNA sequence assembly program. Genome Res. 1999;9(9):868-77.

31. Parra G, Bradnam K, Korf I. CEGMA: a pipeline to accurately annotate core genes in eukaryotic genomes. Bioinformatics. 2007;23(9):1061-7.

32. Li B, Fillmore N, Bai Y, Collins M, Thomson JA, Stewart R, Dewey CN Evaluation of de novo transcriptome assemblies from RNA-Seq data. Genome Biol. 2014;15(12):553.

33. Jones P, Binns D, Chang H-Y, Fraser M, Li W, McAnulla C, McWilliam H, Maslen J, Mitchell A, Nuka G. InterProScan 5: genome-scale protein function classification. Bioinformatics. 2014;30(9):1236-40.

34. Borkotoky S, Saravanan V, Jaiswal A, Das B, Selvaraj S, Murali A, Lakshmi P. The Arabidopsis stress responsive gene database. Int J Plant Genomics. 2013:2013:949564

35. Alter S, Bader KC, Spannagl M, Wang Y, Bauer E, Schön C-C, Mayer KF. DroughtDB: an expert-curated compilation of plant drought stress genes and their homologs in nine species. Database. 2015;2015:bav046.
36. Pfaffl MW. A new mathematical model for relative quantification in real-time RT-PCR. Nucleic Acids Res. 2001;29(9):e45.

37. Da Maia LC, Palmieri DA, De Souza VQ, Kopp MM, de Carvalho FIF. Costa de Oliveira a: SSR locator: tool for simple sequence repeat discovery integrated with primer design and PCR simulation. Int J Plant Genomics. 2008;41:2696.

38. Choy JY, Boon PL, Bertin N, Fullwood MJ. A resource of ribosomal RNAdepleted RNA-Seq data from different normal adult and fetal human tissues. Sci Data. 2015;2:150063. doi:10.1038/sdata.2015.63.

39. Haznedaroglu BZ, Reeves D, Rismani-Yazdi H, Peccia J. Optimization of de novo transcriptome assembly from high-throughput short read sequencing data improves functional annotation for non-model organisms. BMC Bioinf. 2012;13(1):1.

40. Li W, Godzik A. Cd-hit: a fast program for clustering and comparing large sets of protein or nucleotide sequences. Bioinformatics. 2006;22(13):1658-9.

41. Jain M, Srivastava PL, Verma M, Ghangal R, Garg R. De novo transcriptome assembly and comprehensive expression profiling in Crocus sativus to gain insights into apocarotenoid biosynthesis. Sci Rep. 2016;6:22456. doi:10.1038/ srep22456.

42. Wang C, Grohme MA, Mali B, Schill RO, Frohme M. Towards decrypting cryptobiosis - analyzing anhydrobiosis in the tardigrade Milnesium Tardigradum using transcriptome sequencing. PLoS One. 2014;9(3):e92663.

43. Salzberg SL, Phillippy AM, Zimin A, Puiu D, Magoc T, Koren S, Treangen TJ, Schatz MC, Delcher AL, Roberts M. GAGE: a critical evaluation of genome assemblies and assembly algorithms. Genome Res. 2012;22(3):557-67.

44. Moreton J, Dunham SP, Emes RD. A consensus approach to vertebrate de novo transcriptome assembly from RNA-seq data: assembly of the duck (Anas platyrhynchos) transcriptome. Front Genet. 2014;5:190.

45. Ryan DE, Pepper AE, Campbell L. De novo assembly and characterization of the transcriptome of the toxic dinoflagellate Karenia brevis. BMC Genomics. 2014;15(1):1.

46. Azim MK, Khan IA, Zhang Y. Characterization of mango (Mangifera indica L.) transcriptome and chloroplast genome. Plant Mol Biol. 2014;85(1-2):193-208.

47. Hornett EA, Wheat CW. Quantitative RNA-Seq analysis in non-model species: assessing transcriptome assemblies as a scaffold and the utility of evolutionary divergent genomic reference species. BMC Genomics. 2012;13(1):361.

48. Van Belleghem SM, Roelofs D, Van Houdt J, Hendrickx F. De novo transcriptome assembly and SNP discovery in the wing polymorphic salt marsh beetle Pogonus chalceus (Coleoptera, Carabidae). PLoS One. 2012;7(8):e42605

49. Riesgo A, Andrade SC, Sharma PP, Novo M, Pérez-Porro AR, Vahtera V, González VL, Kawauchi GY, Giribet G. Comparative description of ten transcriptomes of newly sequenced invertebrates and efficiency estimation of genomic sampling in non-model taxa. Front Zool. 2012;9(1):1-24.

50. Finseth FR, Harrison RG. A comparison of next generation sequencing technologies for transcriptome assembly and utility for RNA-Seq in a non-model bird. PLoS One. 2014;9(10):e108550

51. Ghaffari N, Arshad O, Jeong H, Thiltges J, Criscitiello M, Yoon B-J, Datta A, Johnson C: Examining de novo transcriptome assemblies via a quality assessment pipeline. 2015

52. Schmitz-Linneweber C, Small I. Pentatricopeptide repeat proteins: a socket set for organelle gene expression. Trends Plant Sci. 2008:13(12):663-70.

53. Manna S. An overview of pentatricopeptide repeat proteins and their applications. Biochimie. 2015;113:93-9.

54. Jiang S-C, Mei C, Liang S, Yu Y-T, Lu K, Wu Z, Wang X-F, Zhang D-P. Crucial roles of the pentatricopeptide repeat protein SOAR1 in Arabidopsis response to drought, salt and cold stresses. Plant Mol Biol. 2015;88(4-5):369-85.

55. Kobe B, Kajava AV. The leucine-rich repeat as a protein recognition motif. Curr Opin Struct Biol. 2001;11(6):725-32

56. Stirnimann CU, Petsalaki E, Russell RB, Müller CW. WD40 proteins propel cellular networks. Trends Biochem Sci. 2010;35(10):565-74.

57. Tatusov RL, Fedorova ND, Jackson JD, Jacobs AR, Kiryutin B, Koonin EV, Krylov DM, Mazumder R, Mekhedov SL, Nikolskaya AN. The COG database: an updated version includes eukaryotes. BMC Bioinf. 2003;4(1):1.

58. Consortium GO. The gene ontology (GO) database and informatics resource. Nucleic Acids Res Suppl. 2004;32:D258-61.

59. Torre S, Tattini M, Brunetti C, Fineschi S, Fini A, Ferrini F, Sebastiani F. RNA-seq analysis of Quercus Pubescens leaves: de novo transcriptome assembly, annotation and functional markers development. PLoS One 2014:9(11):e112487.

60. Blanca J, Cañizares J, Roig C, Ziarsolo P, Nuez F, Picó B. Transcriptome characterization and high throughput SSRs and SNPs discovery in Cucurbito pepo (Cucurbitaceae). BMC Genomics. 2011;12(1):1. 
61. Kanehisa M, Araki M, Goto S, Hattori M, Hirakawa M, Itoh M, Katayama T, Kawashima S, Okuda S, Tokimatsu T. KEGG for linking genomes to life and the environment. Nucleic Acids Res Suppl. 2008;36:D480-4.

62. Wang $H$, Wang $H$, Shao $H$, Tang $X$. Recent advances in utilizing transcription factors to improve plant abiotic stress tolerance by transgenic technology. Front Plant Sci. 2016;7:67. doi:10.3389/fpls.2016.00067.

63. Vom Endt D, Kijne JW, Memelink J. Transcription factors controlling plant secondary metabolism: what regulates the regulators? Phytochemistry. 2002;61(2):107-14.

64. Ariel FD, Manavella PA, Dezar CA, Chan RL. The true story of the HD-zip family. Trends Plant Sci. 2007;12(9):419-26.

65. Rushton DL, Tripathi P, Rabara RC, Lin J, Ringler P, Boken AK, Langum TJ, Smidt L, Boomsma DD, Emme NJ. WRKY transcription factors: key components in abscisic acid signalling. Plant Biotechnol J. 2012;10(1):2-11.

66. Redillas MC, Jeong JS, Kim YS, Jung H, Bang SW, Choi YD, Ha SH, Reuzeau C, $\mathrm{Kim}$ JK. The overexpression of OsNAC9 alters the root architecture of rice plants enhancing drought resistance and grain yield under field conditions. Plant Biotechnol J. 2012;10(7):792-805.

67. Castilhos G, Lazzarotto F, Spagnolo-Fonini L, Bodanese-Zanettini MH, Margis-Pinheiro M. Possible roles of basic helix-loop-helix transcription factors in adaptation to drought. Plant Sci. 2014;223:1-7.

68. Shao $H$, Wang $H$, Tang $X$. NAC transcription factors in plant multiple abiotic stress responses: progress and prospects. Front Plant Sci. 2015;6:902. doi:10.3389/fpls.2015.00902.

69. Wang $Y H$, Irving HR. Developing a model of plant hormone interactions. Plant Signal Behav. 2011;6(4):494-500.

70. Cramer GR, Urano K, Delrot S, Pezzotti M, Shinozaki K. Effects of abiotic stress on plants: a systems biology perspective. BMC Plant Biol. 2011;11(1):163

71. Mano Y, Nemoto K. The pathway of auxin biosynthesis in plants. J Exp Bot 2012;63:2853-72.

72. Agati G, Azzarello E, Pollastri S, Tattini M. Flavonoids as antioxidants in plants: location and functional significance. Plant Sci. 2012;196:67-76.

73. Winkel-Shirley B. Biosynthesis of flavonoids and effects of stress. Curr Opin Plant Biol. 2002;5(3):218-23.

74. Nadernejad N, Ahmadimoghadam A, Hossyinifard J, Poorseyedi S. Effect of different rootstocks on PAL activity and phenolic compounds in flowers, leaves, hulls and kernels of three pistachio (Pistacia vera L.) cultivars. Trees. 2013;27(6):1681-9.

75. Pi E, Qu L, Hu J, Huang Y, Qiu L, Lu H, Jiang B, Liu C, Peng T, Zhao Y. Mechanisms of soybean roots' tolerances to salinity revealed by proteomic and phosphoproteomic comparisons between two cultivars. Mol Cell Proteomics. 2016;15(1):266-88.

76. Kang J-H, McRoberts J, Shi F, Moreno JE, Jones AD, Howe GA. The flavonoid biosynthetic enzyme chalcone isomerase modulates terpenoid production in glandular trichomes of tomato. Plant Physiol. 2014;164(3):1161-74.

77. Wu X, Prior RL. Identification and characterization of anthocyanins by highperformance liquid chromatography-electrospray ionization-tandem mass spectrometry in common foods in the United States: vegetables, nuts, and grains. J Agric Food Chem. 2005;53(8):3101-13.

78. Shinozaki K, Yamaguchi-Shinozaki K. Gene networks involved in drought stress response and tolerance. J Exp Bot. 2007;58(2):221-7.

79. Sinha AK, Jaggi M, Raghuram B, Tuteja N. Mitogen-activated protein kinase signaling in plants under abiotic stress. Plant Signal Behav. 2011;6(2):196-203.

80. Teige M, Scheikl E, Eulgem T, Dóczi R, Ichimura K, Shinozaki K, Dangl JL, Hirt H. The MKK2 pathway mediates cold and salt stress signaling in Arabidopsis. Mole cell. 2004;15(1):141-52.

81. Yu L, Nie J, Cao C, Jin Y, Yan M, Wang F, Liu J, Xiao Y, Liang Y, Zhang W. Phosphatidic acid mediates salt stress response by regulation of MPK6 in Arabidopsis Thaliana. New Phytol. 2010;188(3):762-73.

82. Ji H, Pardo JM, Batelli G, Van Oosten MJ, Bressan RA, Li X. The salt overly sensitive (SOS) pathway: established and emerging roles. Mol Plant. 2013;6(2):275-86.

83. Xiong L, Schumaker KS, Zhu J-K. Cell signaling during cold, drought, and salt stress. Plant Cell. 2002;14:S165-83.

84. You J, Chan Z. ROS regulation during abiotic stress responses in crop plants. Front Plant Sci. 2015;6:1092. doi:10.3389/fpls.2015.01092.

85. Kumar K, Kumar M, Kim S-R, Ryu H, Cho Y-G. Insights into genomics of salt stress response in rice. Rice. 2013;6(1):27.

86. Xiong L, Zhu J-K. Regulation of abscisic acid biosynthesis. Plant Physiol. 2003;133(1):29-36
87. Zheng L, Meng Y, Ma J, Zhao X, Cheng T, Ji J, Chang E, Meng C, Deng N, Chen $\mathrm{L}$. Transcriptomic analysis reveals importance of ROS and phytohormones in response to short-term salinity stress in Populus tomentosa. Front Plant Sci. 2015;6:678.

88. Panahi B. Effects of osmotic and salt stresses on water relation parameters of pistachio seedlings. J Plant Ecophysiol. 2009;1:1-8.

89. Zörb C, Geilfus C-M, Mühling KH, Ludwig-Müller J. The influence of salt stress on $A B A$ and auxin concentrations in two maize cultivars differing in salt resistance. J Plant Physiol. 2013;170(2):220-4.

90. Amjad M, Akhtar J, Anwar-ul-Haq M, Yang A, Akhtar SS, Jacobsen S-E. Integrating role of ethylene and $\mathrm{ABA}$ in tomato plants adaptation to salt stress. Sci Hortic. 2014;172:109-16.

91. Singh A, Jha SK, Bagri J, Pandey GK. ABA inducible rice protein phosphatase $2 C$ confers ABA insensitivity and abiotic stress tolerance in Arabidopsis. PLoS One. 2015:10(4):e0125168.

92. Wu Y-H, Wang T, Wang K, Liang Q-Y, Bai Z-Y, Liu Q-L, Pan Y-Z, Jiang B-B, Zhang L. Comparative analysis of the chrysanthemum leaf transcript profiling in response to salt stress. PLoS One. 2016;11(7):e0159721.

93. Peng Z, He S, Sun J, Pan Z, Gong W, Lu Y, Du X. Na+ compartmentalization related to salinity stress tolerance in upland cotton (Gossypium hirsutum) seedlings. Sci Rep. 2016;6:34548. doi:10.1038/srep34548.

94. Vivek PJ, Tuteja N, Soniya EV. CDPK1 from ginger promotes salinity and drought stress tolerance without yield penalty by improving growth and photosynthesis in Nicotiana Tabacum. PLoS One. 2013;8(10):e76392.

95. Arora A, Kundu S, Dilbaghi N, Sharma I, Tiwari R. Population structure and genetic diversity among Indian wheat varieties using microsatellite (SSR) markers. Aust J Crop Sci. 2014;8(9):1281.

96. Gao Z, Wu J, Liu Z, Wang L, Ren H, Shu Q. Rapid microsatellite development for tree peony and its implications. BMC Genomics. 2013;14(1):1.

97. Ma X, Wang P, Zhou S, Sun Y, Liu N, Li X, Hou Y. De novo transcriptome sequencing and comprehensive analysis of the drought-responsive genes in the desert plant Cynanchum Komarovii. BMC Genomics. 2015;16(1):1.

98. Shi J, Huang S, Zhan J, Yu J, Wang X, Hua W, Liu S, Liu G, Wang H. Genomewide microsatellite characterization and marker development in the sequenced Brassica crop species. DNA Res. 2014;21(1):53-68.

\section{Submit your next manuscript to BioMed Central and we will help you at every step:}

- We accept pre-submission inquiries

- Our selector tool helps you to find the most relevant journal

- We provide round the clock customer support

- Convenient online submission

- Thorough peer review

- Inclusion in PubMed and all major indexing services

- Maximum visibility for your research

Submit your manuscript at www.biomedcentral.com/submit 